\title{
Efficient delivery of small interfering RNAs targeting particular mRNAs into pancreatic cancer cells inhibits invasiveness and metastasis of pancreatic tumors
}

\author{
Keisuke Taniuchi ${ }^{1,2}$, Toshio Yawata ${ }^{3}$, Makiko Tsuboi $^{1}$, Tetsuya Ueba ${ }^{3}$ and Toshiji \\ Saibara ${ }^{1,2}$ \\ ${ }^{1}$ Department of Gastroenterology and Hepatology, Kochi Medical School, Kochi University, Nankoku, Kochi 783-8505, Japan \\ ${ }^{2}$ Department of Endoscopic Diagnostics and Therapeutics, Kochi Medical School, Kochi University, Nankoku, Kochi 783-8505, \\ Japan \\ ${ }^{3}$ Department of Neurosurgery, Kochi Medical School, Kochi University, Nankoku, Kochi 783-8505, Japan \\ Correspondence to: Keisuke Taniuchi, email: ktaniuchi@kochi-u.ac.jp
}

Keywords: pancreatic cancer; small interfering RNA; nanoparticles; delivery system; invasion

Received: January 21, $2019 \quad$ Accepted: April 08, $2019 \quad$ Published: April 23, 2019

Copyright: Taniuchi et al. This is an open-access article distributed under the terms of the Creative Commons Attribution License 3.0 (CC BY 3.0), which permits unrestricted use, distribution, and reproduction in any medium, provided the original author and source are credited.

\section{ABSTRACT}

We report the use of small interfering RNAs (siRNAs) against ARHGEF4, CCDC88A, LAMTOR2, MTOR, NUP85, and WASF2 and folic acid (FA)-modified polyethylene glycol (PEG)-chitosan oligosaccharide lactate (COL) nanoparticles for targeting, imaging, delivery, gene silencing, and inhibition of invasiveness and metastasis in an orthotopic xenograft model. In vitro assays revealed that these siRNA-FA-PEG-COL nanoparticles were specifically inserted into pancreatic cancer cells compared to immortalized normal pancreatic epithelial cells and knocked down expression of the corresponding targets in pancreatic cancer cells. Cell motility and invasion were significantly inhibited by adding target siRNA-FA-PEG-COL nanoparticles into the culture medium. In vivo mouse experiments confirmed that when intravenously delivered, these siRNA-FAPEG-COL nanoparticles became incorporated into human pancreatic cancer cells in mouse pancreatic tumors. Little accumulation was seen in the normal pancreas and vital organs. All target siRNA-FA-PEG-COL nanoparticles significantly inhibited retroperitoneal invasion. The siRNA-FA-PEG-COL nanoparticles against LAMTOR2, $m T O R$, and NUP85, which strongly inhibited retroperitoneal invasion and significantly inhibited peritoneal dissemination compared to the other nanoparticles, improved prognosis of the mice. Our results imply that siRNA-FA-PEG-COL nanoparticles against these six targets could have great potential as biodegradable drug carriers. In particular, siRNA nanoparticles against LAMTOR2, mTOR, and NUP85 may hold significant clinical promise.

\section{INTRODUCTION}

Pancreatic ductal adenocarcinoma (PDAC) is a major cause of death from cancer, with approximately a quarter of a million people worldwide dying annually from PDAC [1]. The prognosis is poor, with 1- and 5-year survival rates of only $20 \%$ and $6 \%$, respectively [2]. About half of patients present with metastatic or end-stage disease and $35 \%$ with localized unresectable disease. Among the $20 \%$ with potentially resectable disease, very few will be cured [3], underscoring the need for more effective systemic therapies coupled with targeted agents and strategies that can improve quality of life. The current standard of chemotherapy for newly diagnosed patients with advanced PDAC is either FOLFIRINOX (5-fluorouracil, leucovorin, irinotecan, and oxaliplatin) or gemcitabine/nab-paclitaxel regimens, but the 5-year survival rate is less than 5\% [4]. The gemcitabine/nab-paclitaxel regimen often causes progressive peripheral neuropathy, limiting the duration of therapy [5]. Regarding targeted therapies, genetic analysis 
of PDAC has yielded insights related to altered signaling pathways [6]; however, unlike other cancers, the number of sequenced PDAC genomes is relatively modest. The era of targeted therapies has offered a new avenue to search for more potentially effective strategies.

PDAC is more likely to invade and metastasize at earlier stages compared to other cancers. At the time of diagnosis, $>80 \%$ of patients with PDAC have locally advanced or metastatic disease [7]. However, the mechanism and details of the molecules involved in invasion and metastasis have not yet been clarified, which hinders the development of novel treatments for suppressing the invasion and metastasis of PDAC. We recently reported that insulin-like growth factor-2 mRNAbinding protein 3 (IGF2BP3)-bound messenger RNAs (mRNAs) are localized in cytoplasmic RNA granules that are transported to the membrane protrusions of PDAC cells by a kinesin motor, Kinesin Family Member 20A [8, 9]. Knockdown of IGF2BP3 suppresses the formation of cell protrusions in S2-013 cells, resulting in a round-tooval morphology of these cells [8]. Furthermore, a total of 2,826 IGF2BP3-bound RNAs were identified [8]. From these RNAs, those associated with cell motility, invasion, and/or metastasis were selected by gene ontology analysis [8]. An IGF2BP3-bound mRNA, ADP-ribosylation factor $6(A R F 6)$ is subsequently translated in membrane protrusions; in turn, locally translated ARF6 protein influences formation of additional membrane protrusions and thereby increases the motility and invasiveness of PDAC cells [8]. Knockdown of IGF2BP3-bound mRNAs, including Rho guanine nucleotide exchange factor 4 (ARHGEF4), coiled-coil domain containing 88A $(C C D C 88 A)$, or WAS protein family member 2 (WASF2), with small interfering RNAs (siRNAs) inhibits the in vitro motility and invasiveness of PDAC cells by decreasing cell protrusions [10-12]. Thus, inhibition of IGF2BP3-bound mRNAs associated with cell motility, invasion, and/or metastasis may be effective as targeted molecular therapy, because any such therapy would inhibit the formation of cell protrusions and consequently limit cell motility and invasion of pancreatic cancer cells. This study determined the effect of six siRNAs targeting the mRNA for ARHGEF4, CCDC88A, late endosomal/ lysosomal adaptor, MAPK and MTOR activator 2 (LAMTOR2), mechanistic target of rapamycin kinase (mTOR), nucleoporin 85 (NUP85), and WASF2 on in vivo invasiveness and metastasis.

RNA nanotechnology using synthetic siRNAs has recently emerged as a method for delivery of highly promising new classes of drugs to treat human diseases. However, siRNA is highly anionic and does not readily diffuse across membrane barriers [13]. One way to enhance the delivery of siRNA to the site of action is development of a suitable delivery platform with characteristics that enable biocompatibility, a high loading capacity, protection of siRNA during transport, and high targeting ability [14]. Also, because siRNA has no functional moiety targeted to the sites of interest and its negative charge leads to poor cellular uptake owing to the electrostatic repulsion between siRNA and the cell membrane [15], such targeted delivery systems require a ligand-receptor pair that is specifically found in cancer cells. Folic acid (FA), a synthetic oxidized form of folate, has been widely used as a ligand conjugate in various cancer targeting materials [16, 17]. We previously reported that systemically administered tumortargeting siRNA/FA-poly(ethylene glycol)-chitosan oligosaccharide lactate (FA-PEG-COL) nanoparticles are vital for delivery of siRNA to ovarian cancer site(s) in $\mathrm{BALB} / \mathrm{c}$ mice bearing ovarian cancer tumor xenografts [18]. We demonstrated the uptake of siRNA/FA-PEGCOL nanoparticles into ovarian cancer cells via receptormediated endocytosis [18].

The present study shows the potential utility of an siRNA delivery system with FA-PEG-COL nanoparticles conjugated to six types of siRNAs targeting the mRNA for ARHGEF4, CCDC88A, LAMTOR2, mTOR, NUP85, and $W A S F 2$ as targeted PDAC gene therapy.

\section{RESULTS}

\section{Physical characterization of siRNA-FA-PEG- COL nanoparticles}

FA was linked to COL using hetero-bifunctional PEG. Matrix assisted laser desorption/ionization time-offlight mass spectrometry (MALDI-TOF MS) was used to verify the conjugation of FA to PEG. Consistent with a previous report [18], the mass/charge $(\mathrm{m} / \mathrm{z})$ values of FAPEG and FA-PEG-COL were 3699 and 3652, respectively (Figure $1 \mathrm{~A}$ ). The $\mathrm{m} / \mathrm{z}$ value was not altered by adding COL (Figure 1A). The size of FA-PEG-COL was analyzed by scanning electron microscope (SEM). SEM images showed that the size of FA-PEG-COL was about $80 \mathrm{~nm}$ (Figure 1B).

\section{Insertion of siRNA-FA-PEG-COL nanoparticles into S2-013 and HPNE cells}

Alexa 488-labeled scrambled control siRNA-FAPEG-COL nanoparticles were added to the culture media of S2-013 cells and cultured for $24 \mathrm{~h}$. Flow cytometry data showed cellular uptake of scrambled control siRNA-FAPEG-COL nanoparticles into S2-013 cells (Figure 2A). Confocal microscopy showed that abundant scrambled control siRNA-FA-PEG-COL nanoparticles were present in the cytoplasm, whereas HPNE cells displayed a weak signal (Figure 2B), strongly suggesting that the prepared siRNA-FA-PEG-COL nanoparticles were not inserted into HPNE cells.

Alexa 488-labeled FA-PEG-COL nanoparticles, Alexa 488-labeled scrambled control siRNA-COL, 
and Alexa 488-labeled scrambled control siRNA-FAPEG-COL nanoparticles were cultured with S2-013 cells for $24 \mathrm{~h}$, and confocal microscopy was carried out (Figure 2C). FA-PEG-COL nanoparticles and scrambled control siRNA-FA-PEG-COL nanoparticles were taken up into S2-013 cells more abundantly than scrambled control siRNA-COL, indicating that FA functioned in increasing the cellular uptake of the particles.

\section{Effects of siRNA-FA-PEG-COL nanoparticles on silencing of the target mRNAs in PDAC cells}

FA receptor expression was confirmed in $\mathrm{S} 2-$ 013, PANC-1, and HPNE cells by immunoblotting and immunocytochemical analyses. Immunoblotting showed almost the same level of FA receptor expression in S2-013, PANC-1, and HPNE cells (Figure 3A). To determine whether scrambled control siRNA-FAPEG-COL nanoparticles were bound to FA receptors, immunocytochemistry was performed in S2-013 and HPNE cells (Figure 3B, 3C). Alexa 488-labeled scrambled control siRNA-FA-PEG-COL nanoparticles were added to the culture media of S2-013 and HPNE cells and cultured for $24 \mathrm{~h}$. Scrambled control siRNA-FA-PEG-COL nanoparticles were taken up into the cytoplasm and FA receptors were mainly localized in the cytoplasm of S2013 cells. A portion of the particles and FA receptors was co-localized in granules in the cytoplasm of S2-013 cells, whereas few co-localization of scrambled control siRNAFA-PEG-COL nanoparticles and FA receptors was seen in HPNE cells. These results suggested that scrambled control siRNA-FA-PEG-COL nanoparticles bound to FA receptors were inserted into $\mathrm{S} 2-013$ cells.

Representative semi-quantitative reverse transcription-PCR (RT-PCR) for CCDC88A and WASF2 showed that prepared CCDC88A siRNA-FA-PEGCOL nanoparticles and WASF2 siRNA-FA-PEG-COL nanoparticles knocked down the expression of the
A
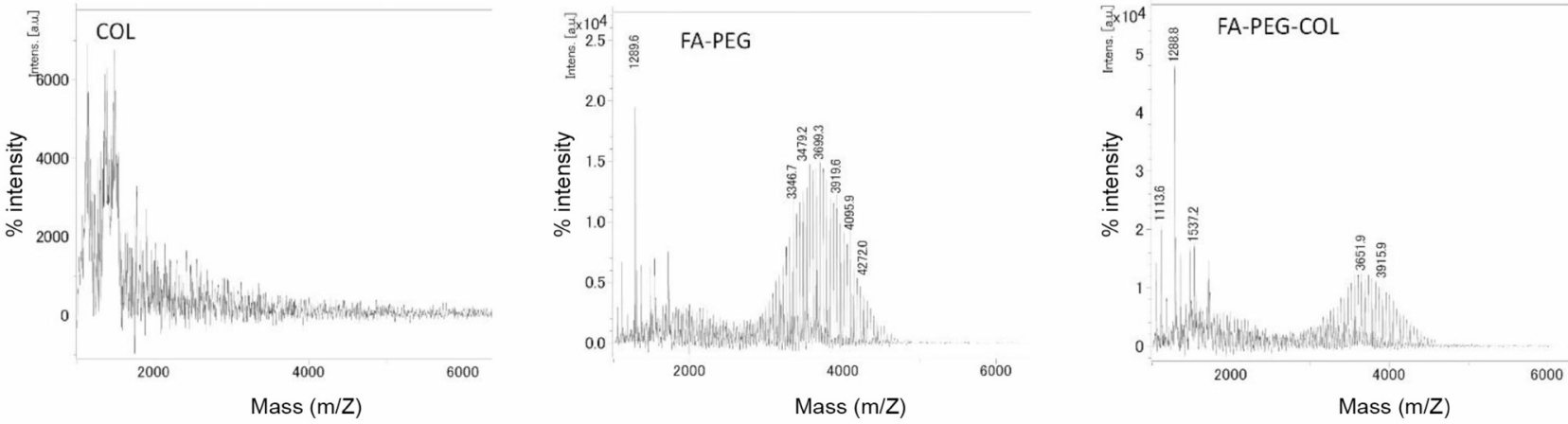

B
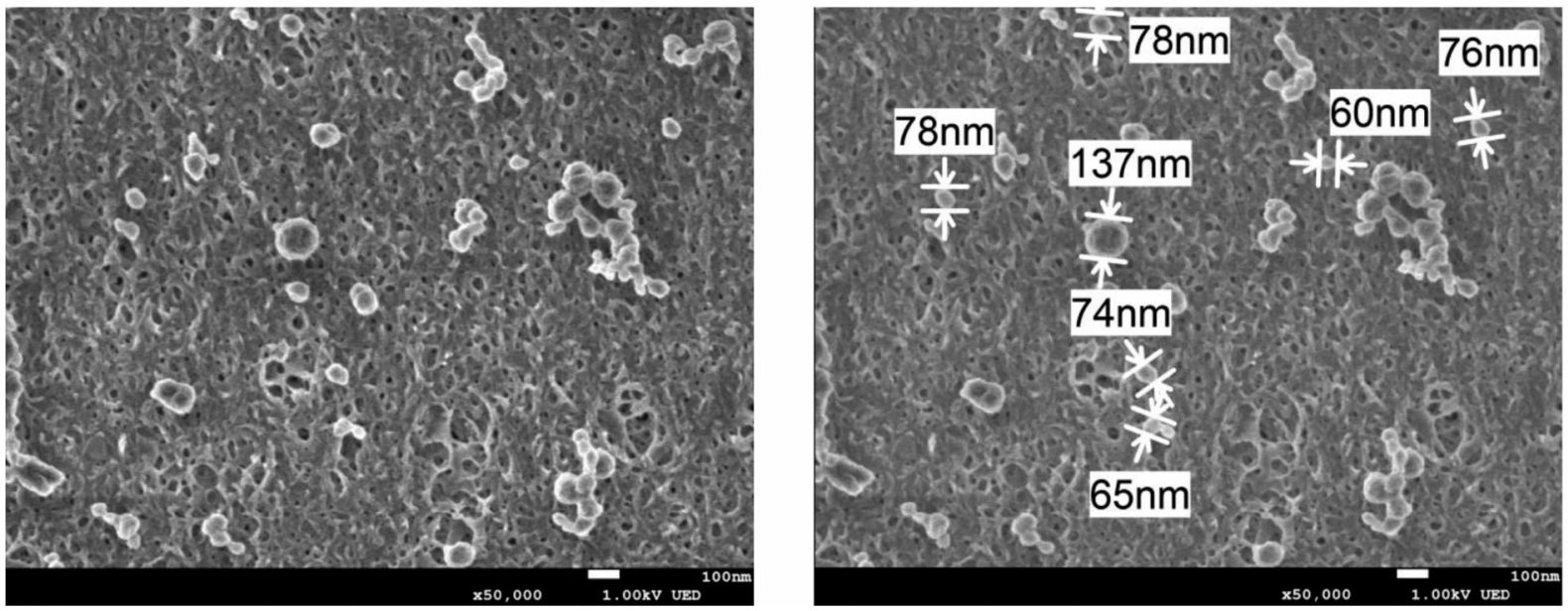

Figure 1: Characterization of siRNA conjugated to FA-PEG-COL. (A) MALDI-TOF Mass analysis of FA-PEG and FAPEG-COL. Data are representative of three independent experiments. (B) SEM images of FA-PEG-COL. Scale bars, $100 \mathrm{~nm}$. Data are representative of three independent experiments. 

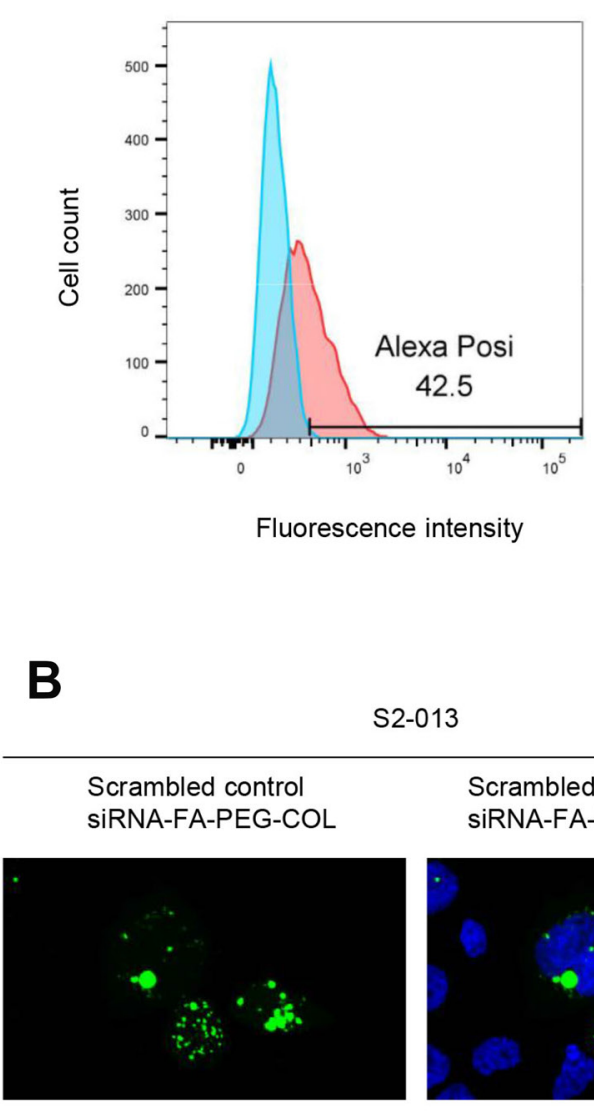

S2-013

HPNE
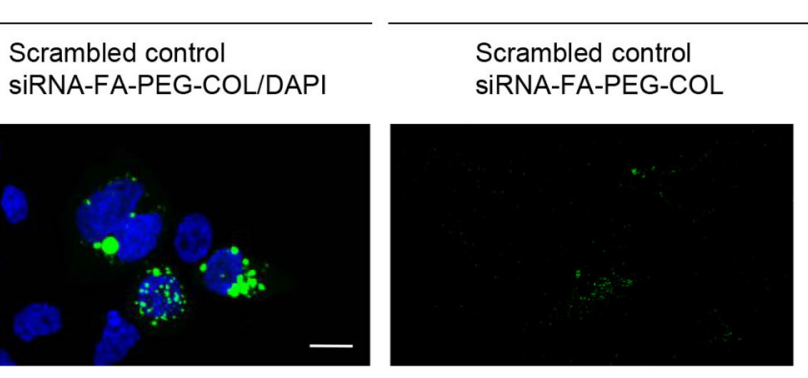

Scrambled control siRNA-FA-PEG-COL/DAPI
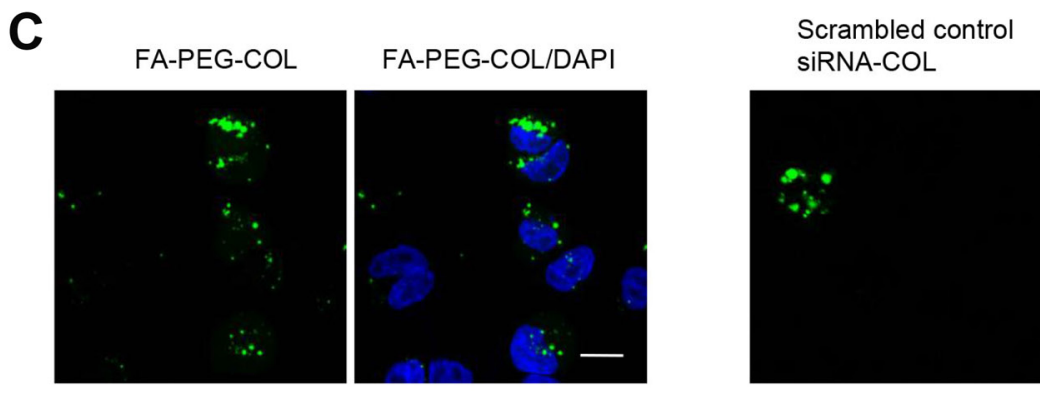

Scrambled control SiRNA-COL/DAPI

Scrambled control

Scrambled control

SiRNA-FA-PEG-COL

SIRNA-FA-PEG-COL/DAPI
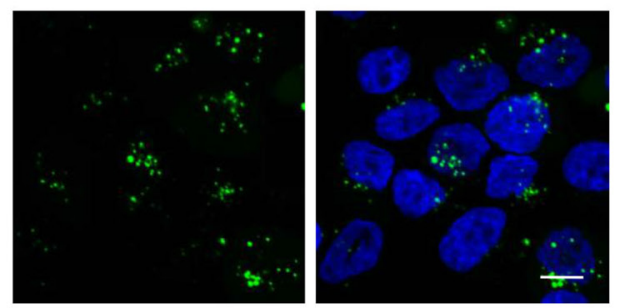

Figure 2: Insertion of siRNA-FA-PEG-COL nanoparticles into S2-013 and HPNE cells. (A) Representative flow cytometry data of Alexa 488-labeled scrambled control siRNA-FA-PEG-COL nanoparticles inserted into S2-013 cells. (B) Confocal immunofluorescence microscopic images of scrambled control siRNA-FA-PEG-COL nanoparticles (green) in S2-013 and HPNE cells. Blue, DAPI staining. Scale bars, $10 \mu \mathrm{m}$. (C) Confocal immunofluorescence microscopic images of FA-PEG-COL nanoparticles (green), scrambled control siRNA-COL (green), and scrambled control siRNA-FA-PEG-COL nanoparticles (green) in S2-013 cells. Blue, DAPI staining. Scale bars, $10 \mu \mathrm{m}$. 
A

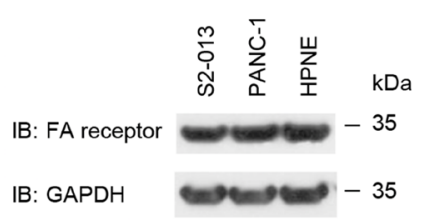

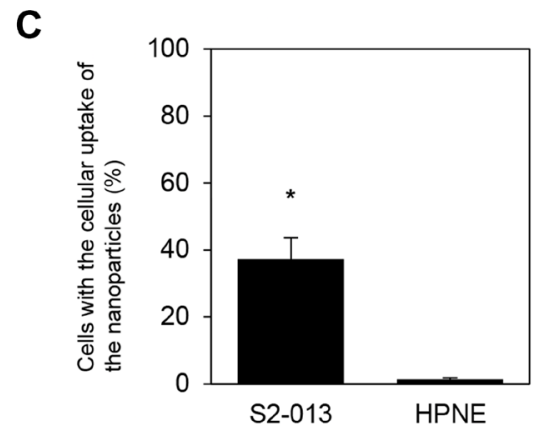

B

S2-013
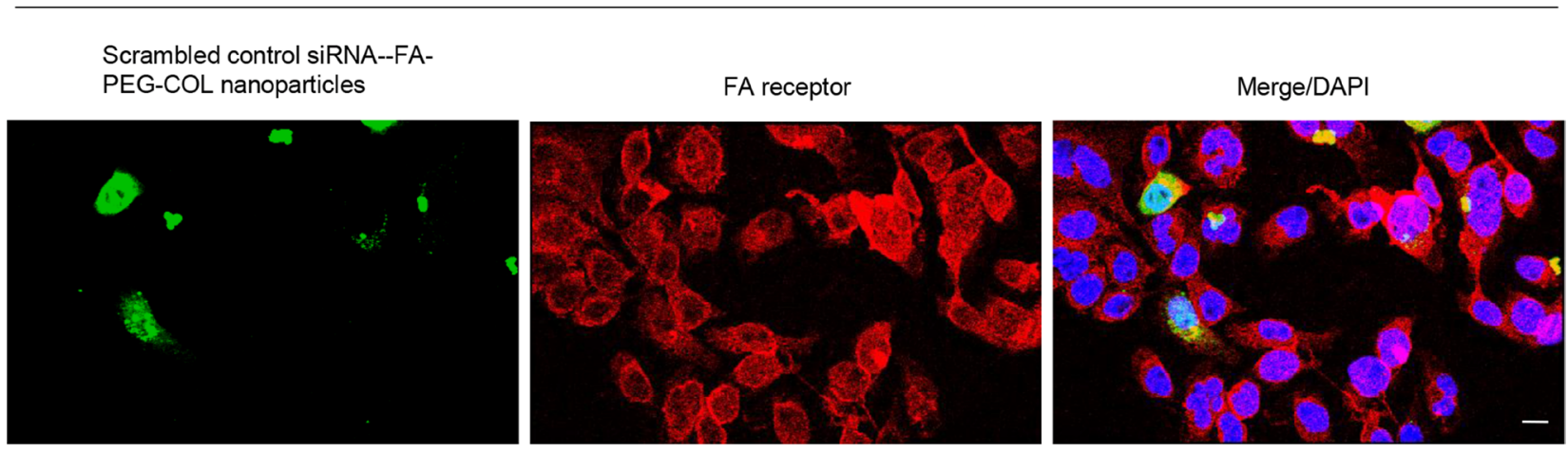

HPNE

Scrambled control siRNA--FAPEG-COL nanoparticles

FA receptor

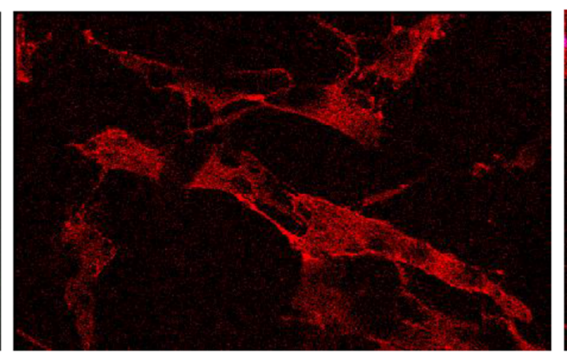

Merge/DAPI

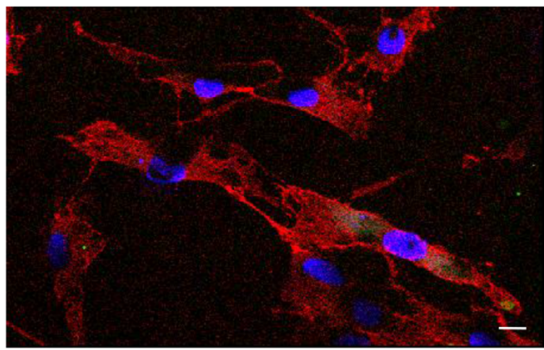

D

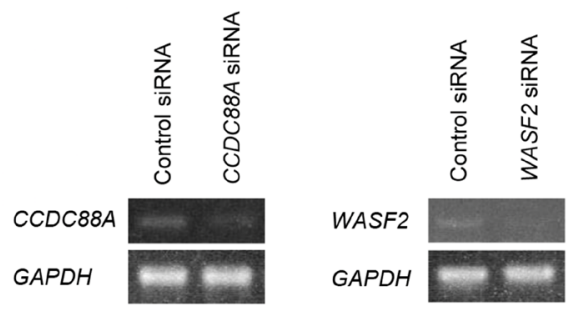

E

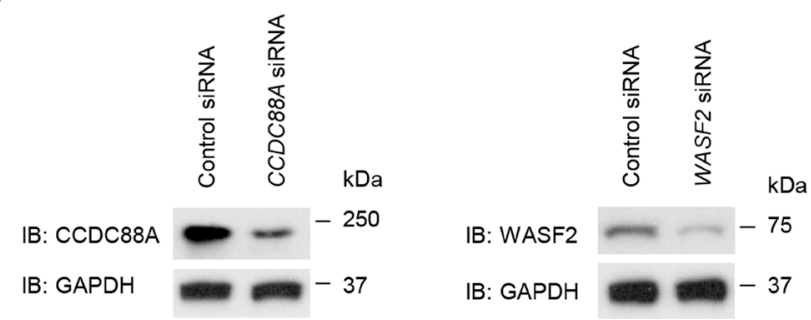

Figure 3: Effect of target siRNA-FA-PEG-COL nanoparticles on silencing of the targets in PDAC lines. (A) Western blotting was performed using anti-FA receptor antibody in S2-013, PANC-1 and HPNE cells. Data are representative of three independent experiments. (B) Confocal immunofluorescence microscopic images of scrambled control siRNA-FA-PEG-COL nanoparticles (green) and FA receptors (red) in S2-013 and HPNE cells. Blue, DAPI staining. Scale bars, $10 \mu \mathrm{m}$. (C) Quantification of the data shown in Figure 3B; the values represent the number of cells with the cellular uptake of scrambled control siRNA-FA-PEG-COL nanoparticles. All cells in four visual fields per group were scored. Data are derived from three independent experiments. Columns, mean; bars, standard deviation (SD). ${ }^{*} p<0.001$ (Student's $t$-test). (D, E) Semi-quantitative RT-PCR (D) and Western blotting (E) for CCDC88A and WASF2 in S2-013 cells incubated with scrambled control siRNA-FA-PEG-COL nanoparticles, CCDC88A siRNA-FA-PEG-COL nanoparticles, or WASF2 siRNAFA-PEG-COL nanoparticles. Data are representative of three independent experiments. 
corresponding mRNAs in S2-013 cells after incubation for $48 \mathrm{~h}$, whereas scrambled control siRNA-FA-PEGCOL nanoparticles did not (Figure 3D). Additionally, Western blotting for CCDC88A and WASF2 showed that $C C D C 88 A$ siRNA-FA-PEG-COL nanoparticles and WASF2 siRNA-FA-PEG-COL nanoparticles downregulated expression of the corresponding protein in S2-013 cells, whereas control siRNA-FA-PEG-COL nanoparticles did not (Figure 3E). Thus, CCDC88A siRNA-FA-PEG-COL nanoparticles and WASF2 siRNAFA-PEG-COL nanoparticles specifically reduced the expression of CCDC88A and WASF2, respectively, in S2013 cells. We confirmed that other siRNA-FA-PEG-COL nanoparticles against mRNAs for ARHGEF4, LAMTOR2, $m T O R$, and NUP85 down-regulated expression of the corresponding mRNA and protein (data not shown).

\section{Effects of knockdown of the target mRNAs on cell motility and invasion in vitro}

We previously reported that ARHGEF4, CCDC88A, and WASF2 are not associated with cell growth in PDAC cells [10-12]. We confirmed that suppression of LAMTOR2, mTOR, and NUP85 using commercial siRNA oligos also did not affect cell growth in an in vitro MTT assay in S2-013 and PANC-1 cells (data not shown). To determine whether knockdown of the target mRNAs using siRNA-FA-PEG-COL nanoparticles affected the motility and invasiveness of PDAC cells, in vitro motility and invasion assays were performed. Suppression of CCDC88A and WASF2 by adding the corresponding siRNA-FA-PEG-COL nanoparticles to the culture medium of S2-013 cells significantly inhibited cell motility in motility assays, compared to S2-013 cells incubated with scrambled control siRNA-FA-PEG-COL nanoparticles (Figure 4A). In two-chamber invasion assays, the corresponding target siRNA-FA-PEG-COL nanoparticles significantly inhibited cell invasion compared to scrambled control siRNA-FA-PEG-COL nanoparticles in S2-013 cells (Figure 4B). We confirmed that other siRNA-FAPEG-COL nanoparticles against mRNAs for ARHGEF4, LAMTOR2, $m T O R$, and NUP85 significantly inhibited cell invasion compared to scrambled control siRNA-FA-PEGCOL nanoparticles in S2-013 cells (Figure 4C).

\section{Delivery of siRNA-FA-PEG-COL nanoparticles to PDAC cells in PDAC tumors of an orthotopic mouse model}

To determine whether siRNA-FA-PEG-COL nanoparticles were delivered to PDAC tissues, we generated an orthotopic mouse model of PDAC by surgical implantation of human S2-013 cells into the pancreas of nude mice [19]. Alexa 647-labeled scrambled control siRNA-COL and Alexa 647-labeled scrambled control siRNA-FA-PEG-COL nanoparticles were intravenously injected into nude mice once per week for six weeks after injection of S2-013 cells into the pancreas. Twentyfour hours after the last injection of the nanoparticles, in vivo imaging studies were performed (Figure 5A). Scrambled control siRNA-COL and scrambled control siRNA-FA-PEG-COL nanoparticles were taken up mainly into the PDAC tumors (Figure 5A). Of note, significant uptake of scrambled control siRNA-FA-PEG-COL nanoparticles into the PDAC tumors was seen compared with accumulation in the PDAC tumors of mice given scrambled control siRNA-COL (Figure 5A).

To confirm the delivery of the siRNA-FA-PEGCOL nanoparticles to PDAC cells within PDAC tumors, Alexa 594-labeled scrambled control siRNA-COL and Alexa 594-labeled scrambled control siRNA-FA-PEGCOL nanoparticles were injected intravenously into the mice administered S2-013 cells 6 weeks before. The mice were perfused $24 \mathrm{~h}$ after intravenous injection of the nanoparticles, and frozen sections of the S2-013derived PDAC tumor tissues that had formed in the mouse pancreas were prepared. Scrambled control siRNA-COL were present in the tumor stroma, and uptake of the nanoparticles into PDAC cells was limited (Figure 5B). In contrast, accumulation of scrambled control siRNA-FAPEG-COL nanoparticles into the S2-013-derived PDAC cells was markedly higher (Figure 5B).

\section{Effects of siRNA-FA-PEG-COL nanoparticles on silencing of the target mRNAs in the orthotopic mouse model of PDAC}

To confirm the knockdown effects of the siRNA-FAPEG-COL nanoparticles in the orthotopic mouse model of PDAC, representative experiments were carried out using siRNA-FA-PEG-COL nanoparticles against CCDC $88 A$ and $W A S F 2$. First, Alexa 647-labeled scrambled control siRNA-FA-PEG-COL nanoparticles, Alexa 647-labeled CCDC88A siRNA-FA-PEG-COL nanoparticles, and Alexa 647-labeled WASF2 siRNA-FA-PEG-COL nanoparticles were intravenously injected into the nude mice 6 weeks after injection of S2-013 cells into the pancreas of the nude mice. Twenty-four hours after injection, ex vivo imaging studies were performed (Figure 6A). Ex vivo images of PDAC tumors in sacrificed mice showed the presence of scrambled control siRNA-FA-PEG-COL nanoparticles, CCDC88A siRNA-FA-PEG-COL nanoparticles, and WASF2 siRNA-FA-PEG-COL nanoparticles at the 24-h time point. In addition, scrambled control siRNA-FAPEG-COL nanoparticles were accumulated in a peritoneal dissemination nodule from PDAC tumors.

To determine the knockdown effect of the siRNAFA-PEG-COL nanoparticles against CCDC88A and $W A S F 2$, mice that were administered S2-013 cells 6 weeks before were perfused $24 \mathrm{~h}$ after intravenous injection of Alexa 647-labeled siRNA-FA-PEG-COL nanoparticles against $C C D C 88 A$ and $W A S F 2$ or Alexa 647-labeled 
scrambled control siRNA-FA-PEG-COL nanoparticles. Frozen tissue sections of the human PDAC tissues derived from S2-013 cells in the mouse pancreas were prepared, and confocal immunocytochemical analysis was performed using the corresponding antibodies against each target of the siRNA-FA-PEG-COL nanoparticles. The levels of protein expression of CCDC88A and WASF2 were higher in PDAC cells that had taken up the siRNA-FA-PEG-COL nanoparticles against CCDC88A and $W A S F 2$ poorly compared to PDAC cells that had efficiently taken up the nanoparticles and those that had taken up scrambled control siRNA-FA-PEG-COL nanoparticles (Figure 6B).

\section{Effects of knockdown of the target mRNAs on invasiveness and metastasis in the orthotopic mouse model of PDAC}

To study the effects of siRNA-FA-PEG-COL nanoparticles against the target mRNAs on invasiveness and metastasis in vivo, the nude mouse model of PDAC established by injection of S2-013 cells into the pancreas was used. Three control groups were included: 1) scrambled control siRNA-FA-PEG-COL nanoparticles, 2) phosphate-buffered saline (PBS) alone, and 3) scrambled control siRNA-COL alone. On day 4 after injection of S2013 cells, mice in each group received the first intravenous injection of nanoparticles or control solutions. All mice received a total of five intravenous injections once a week. Forty-two days after implantation, mice were sacrificed, sections of PDAC tissues, lung, and liver were prepared, and hematoxylin and eosin staining was performed to determine the presence or absence of peritoneal dissemination, and distant liver and lung metastases. In the control groups, $70 \%$ of the S2-013-implanted mice developed extensive peritoneal carcinomatosis (Figure 7A), and all of the mice developed hemorrhagic ascites. Invasion into adjacent organs, such as the spleen, stomach, and colon, was commonly observed in control groups. Hematoxylin and eosin staining of
A

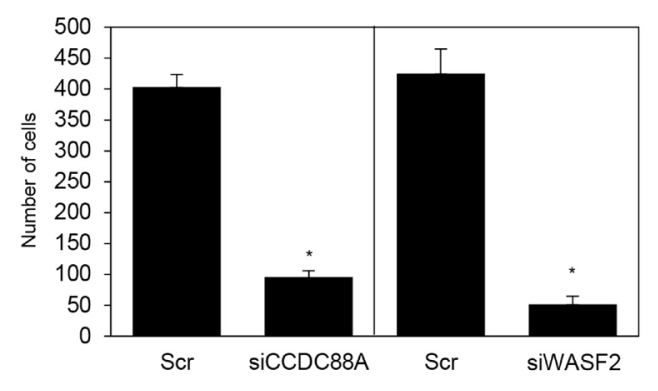

B

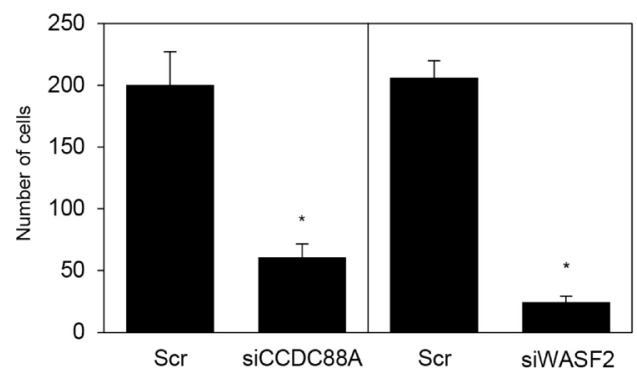

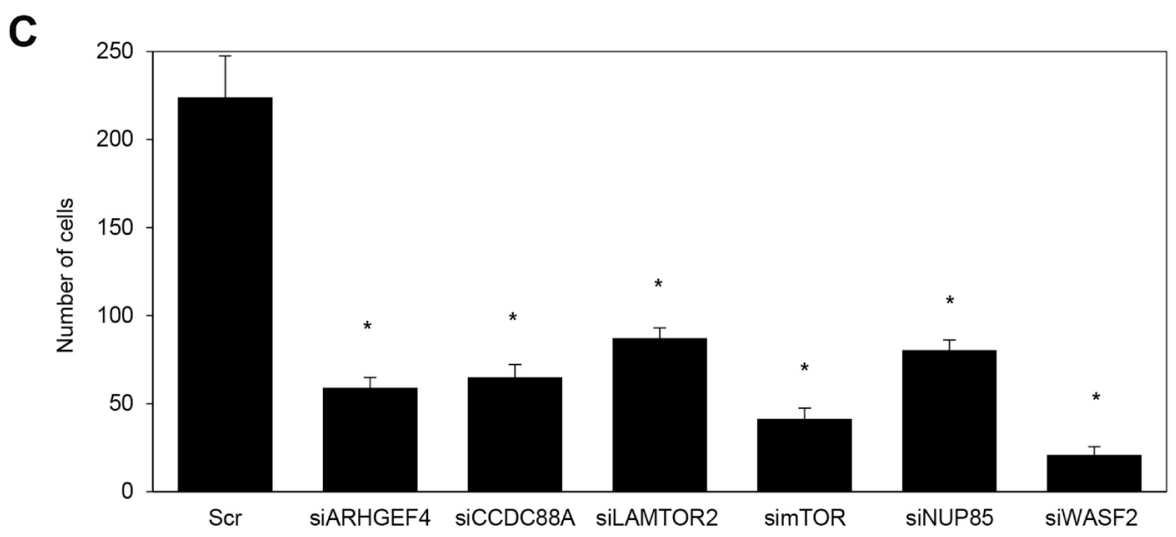

Figure 4: Knockdown effect of the target siRNA-FA-PEG-COL nanoparticles on cell motility and invasion in vitro. (A, B) S2-013 cells were incubated with scrambled control siRNA-FA-PEG-COL nanoparticles (Scr), CCDC88A siRNA-FA-PEG-COL nanoparticles (siCCDC88A), or WASF2 siRNA-FA-PEG-COL nanoparticles (siWASF2). Motility (A) and two-chamber invasion (B) assays were performed. Migrating cells in four fields per group were scored. Data were derived from three independent experiments. Columns, mean; bars, SD. ${ }^{*} p<0.05$ compared to cells incubated with scrambled control siRNA-FA-PEG-COL nanoparticles (Student's $t$-test). (C) S2-013 cells were incubated with scrambled control siRNA-FA-PEG-COL nanoparticles (Scr) and the target siRNA-FA-PEG-COL nanoparticles against mRNAs for ARHGEF4 (siARHGEF4), LAMTOR2 (siLAMTOR2), mTOR (simTOR), and NUP85 (siNUP85). Twochamber invasion assay was performed. Migrating cells in four fields per group were scored. Data were derived from three independent experiments. Columns, mean; bars, standard deviation (SD). ${ }^{*} p<0.05$ compared to cells incubated with scrambled control siRNA-FAPEG-COL nanoparticles (Student's $t$-test). 
representative sections of S2-013-derived PDAC tumors showed adenocarcinoma with regional invasion of the retroperitoneum (Figure 7B). Histologic analysis of liver and lung metastases is shown in Figure 7C and 7D, respectively. Fisher's exact test showed that all target siRNA-FA-PEG-COL nanoparticles significantly inhibited retroperitoneal invasion compared to the control groups (Table 1). Of note, the siRNA-FA-PEG-COL nanoparticles against mRNAs for LAMTOR2, mTOR, and NUP85 strongly inhibited regional invasion of the retroperitoneum and significantly inhibited peritoneal dissemination. siRNA-FA-PEG-COL nanoparticles against CCDC88A, ARHGEF4, LAMTOR2, and WASF2 significantly inhibited lung metastasis compared to the control groups (Table 1). The target siRNA-FA-PEG-COL nanoparticles did not inhibited liver metastasis compared to the control groups.

\section{A}

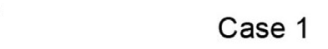

Scrambled control Scrambled control siRNA-FA-PEG-COL siRNA-COL nanoparticles

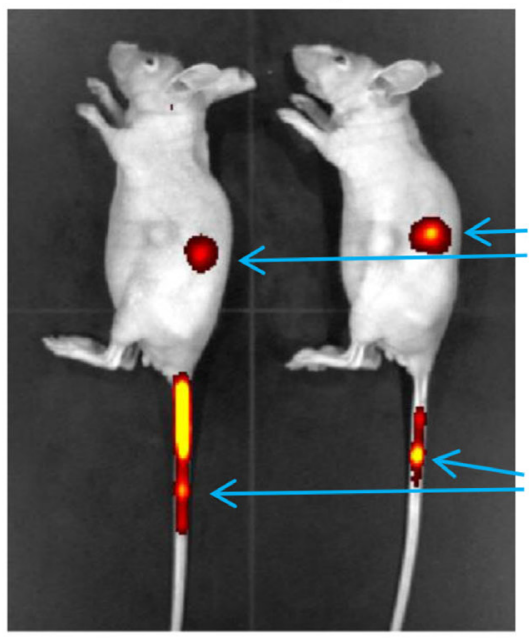

Accumulation in S2-013derived pancreatic cancer tumors

\section{Injection via the tail vein}

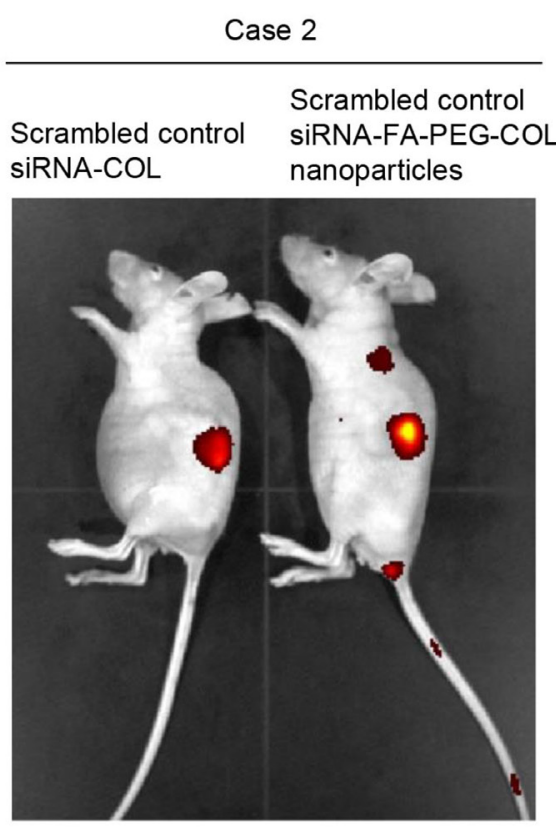

\section{B}

Scrambled control siRNA-COL

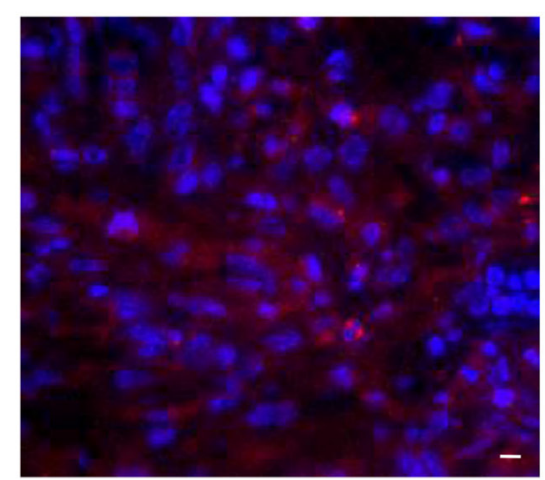

Scrambled control siRNA-FA-PEGCOL nanoparticles

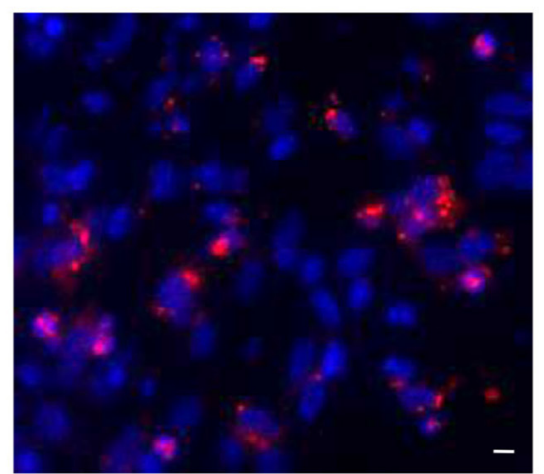

Figure 5: Delivery of siRNA-FA-PEG-COL nanoparticles to PDAC cells in the orthotopic mouse model of PDAC. (A) Whole-body in vivo imaging of the S2-013 tumor-bearing mice after intravenous injection of Alexa 647-labeled scrambled control siRNACOL and Alexa 647-labeled scrambled control siRNA-FA-PEG-COL nanoparticles via the tail vein. Fluorescence intensity of the Alexa 647-labeled nanoparticles, which accumulated in S2-013-derived PDAC tumors, was measured $24 \mathrm{~h}$ after intravenous injection into the mice. (B) The S2-013 tumor-bearing mice were fixed by perfusion $24 \mathrm{~h}$ after intravenous injection of Alexa 647-labeled scrambled control siRNA-COL and Alexa 647-labeled scrambled control siRNA-FA-PEG-COL nanoparticles via the tail vein. Representative confocal immunofluorescence microscopic images of frozen sections of S2-013-derived PDAC tumor tissues from mice showing scrambled control siRNA-COL (red) and the siRNA-FA-PEG-COL nanoparticles (red). Blue, DAPI staining. Scale bars, $10 \mu \mathrm{m}$. 


\section{Disease progression and prognosis}

We observed survival of the mice until 8 weeks after implantation of S2-013 cells. Fisher's exact test showed that mice in control groups had significantly worse survival compared to mice given the siRNA-FAPEG-COL nanoparticles against mRNAs for LAMTOR2, mTOR, and NUP85 (Table 2). In contrast, mice treated with the siRNA-FA-PEG-COL nanoparticles against other mRNAs including ARHGEF4, CCDC88A, NUP85, and $W A S F 2$ had similar survival as control groups (Table 2). None of the surviving mice displayed extensive peritoneal carcinomatosis or hemorrhagic ascites, and the tumors in the pancreas were largely encapsulated (Figure 8A, 8B).
Toxicology study of siRNA-FA-PEG-COL nanoparticles in the orthotopic mouse model of PDAC

The safety of siRNA-FA-PEG-COL nanoparticles was assessed. The degree of hemolysis caused by FAPEG-COL nanoparticles and scrambled control siRNAFA-PEG-COL nanoparticles mixed with diluted mouse blood for $1 \mathrm{~h}$ is shown in Figure 9A. In vitro hemolysis tests were negative in these two groups. The positive control chemical (Triton X-100) caused significantly greater levels of hemolysis compared to scrambled control siRNA-FA and scrambled control siRNA-FA-PEG-COL nanoparticles.
A

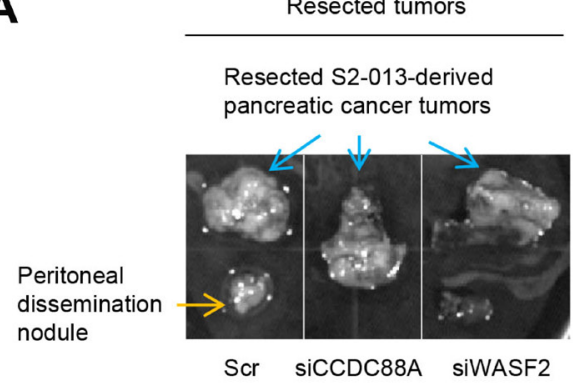

B
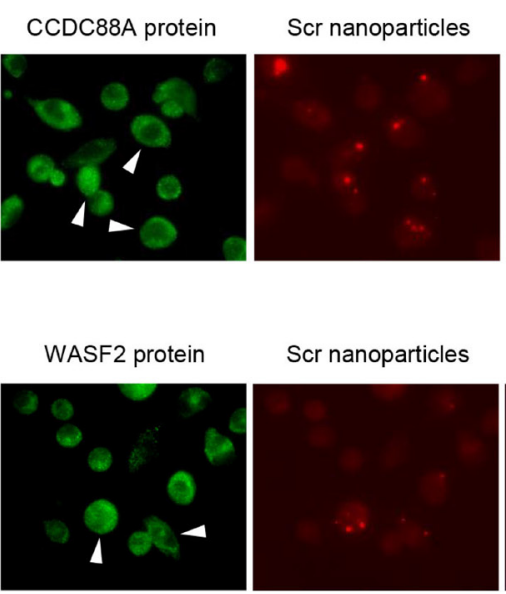

Scr nanoparticles

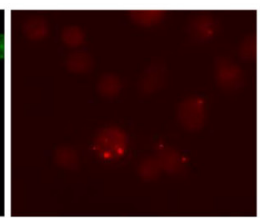

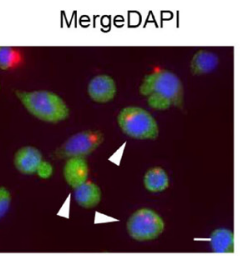

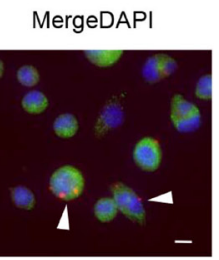

CCD88A protein

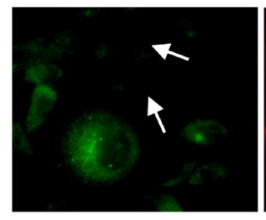

WASF2 protein

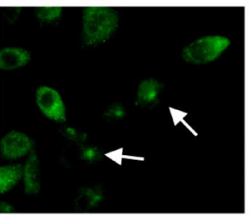

Ex vivo images

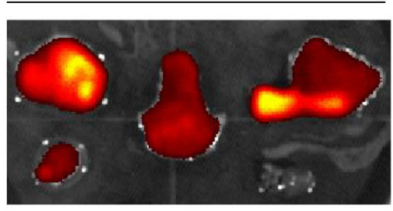

Scr siCCDC88A siWASF2

Figure 6: Effect of target siRNA-FA-PEG-COL nanoparticles on silencing of the targets in the orthotopic mouse model of PDAC. (A) Ex vivo images of the PDAC tumors excised from the S2-013 tumor-bearing mice after intravenous injection of Alexa 647-labeled scrambled control siRNA-FA-PEG-COL nanoparticles (Scr), Alexa 647-labeled CCDC88A siRNA-FA-PEGCOL nanoparticles (siCCDC88A), and Alexa 647-labeled WASF2 siRNA-FA-PEG-COL nanoparticles (siWASF2) via the tail vein. The peritoneal dissemination nodule and mouse heart were excised from the S2-013 tumor-bearing mice after intravenous injection of Alexa 647-labeled scrambled control siRNA-FA-PEG-COL nanoparticles. Fluorescence intensity of the Alexa 647-labeled nanoparticles was measured $24 \mathrm{~h}$ after intravenous injection to the mouse model. (B) The S2-013 tumor-bearing mice were fixed by perfusion $24 \mathrm{~h}$ after intravenous injection of Alexa 647-labeled scrambled control siRNA-FA-PEG-COL nanoparticles (Scr) or Alexa 647-labeled target siRNAFA-PEG-COL nanoparticles against CCDC88A (siCCDC88A) and WASF2 (siWASF2) via the tail vein. Frozen sections of S2-013-derived PDAC tumor tissues were immunocytochemically stained with antibodies corresponding to the target siRNAs (green). Nanoparticles were indicated by red. Representative confocal immunofluorescence microscopic images are shown. Arrows, tumor cells showing suppression of the target proteins by the siRNA-FA-PEG-COL nanoparticles. Arrows, the target siRNA-FA-PEG-COL nanoparticle transfected tumor cells that suppress CCDC88A or WASF2. Arrowheads, the scrambled control siRNA-FA-PEG-COL nanoparticle transfected tumor cells that express CCDC88A and WASF2. Blue, DAPI staining. Scale bars, $10 \mu \mathrm{m}$. 
In addition to hemolysis tests, liver, kidney, and pancreas function tests were performed with blood collected after administration of siRNAs with nanoparticles (Figure 9B). Nude mice (6 weeks old; four animals per group) were given intravenous injections of PBS alone (PBS group), FA-PEG-COL nanoparticles alone (Particle group), scrambled control siRNA-FAPEG-COL nanoparticles (Scrambled control group), or siRNA-FA-PEG-COL nanoparticles against CCDC88A (Target group). Finally, all mice were given a total of five intravenous injections once a week. At week 6, we collected blood from all mice to check liver [aspartate aminotransferase (AST), alanine aminotransferase (ALT), gamma-glutamyl transferase $(\gamma-\mathrm{GT})$, and total bilirubin (T-BIL)], kidney [urea nitrogen (BUN), creatinine (CRE)], and pancreas [amylase (AMY)] functions. No group had abnormal ranges for any of the parameters compared with the PBS group. Histopathological analysis showed no abnormal pathological lesions in the lungs, livers, or kidneys of the mice (data not shown).

\section{DISCUSSION}

PDAC is resistant to conventional chemotherapy and radiation because the cells overexpress genes such as $K$-Ras, $p 16, p 53$, and SMAD4 with different mutations
A

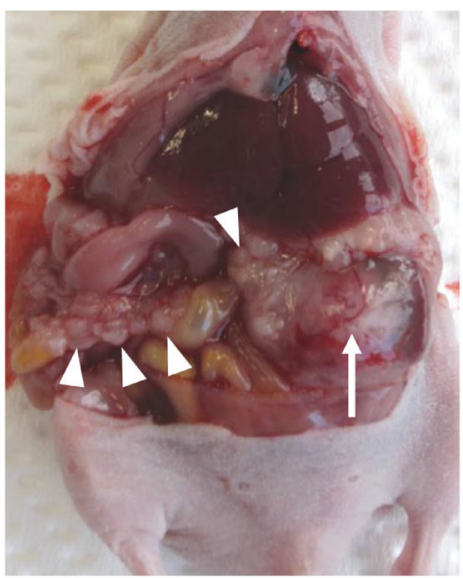

C

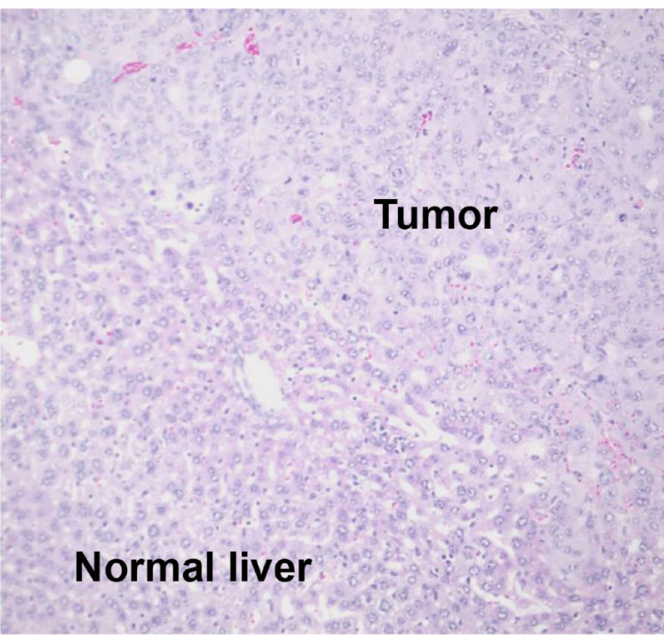

B

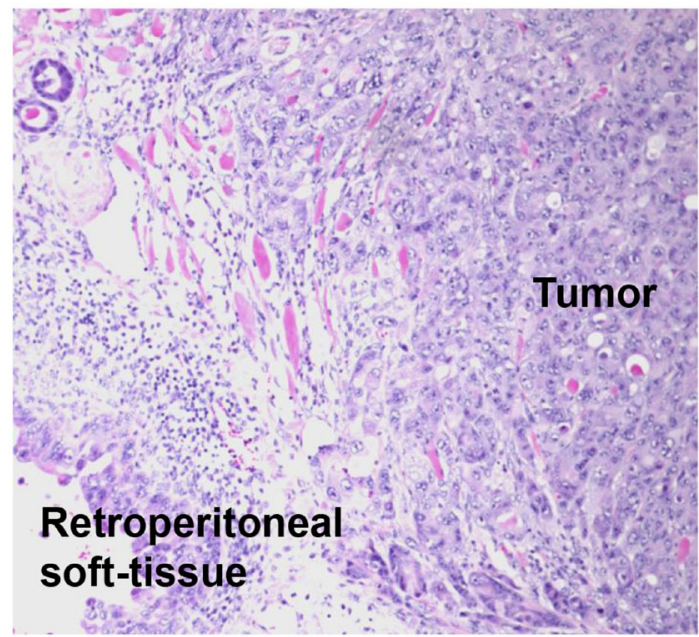

D

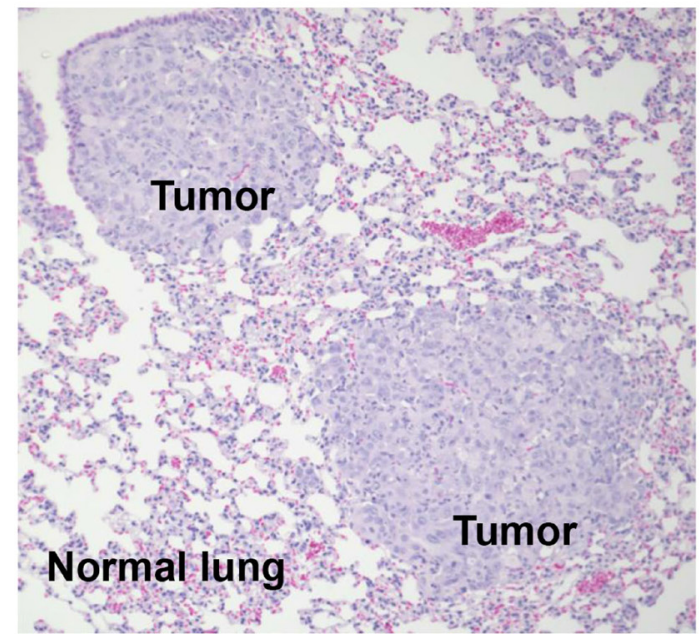

Figure 7: Knockdown effect of target siRNA-FA-PEG-COL nanoparticles on cell motility and invasion in the orthotopic mouse model of PDAC. (A) Development of carcinomatosis in S2-013 tumor-bearing mice treated with scrambled control siRNAFA-PEG-COL nanoparticles. Arrow, primary tumor; arrowheads, dissemination nodules in the abdominal cavity. (B-D) Hematoxylin and eosin staining of representative sections of S2-013-derived PDAC tumor tissues in mice treated with scrambled control siRNA-FA-PEGCOL nanoparticles showing areas of regional invasion of the retroperitoneum (B), and distant metastases to the liver (C) and lung (D). Original magnification: $\times 200$ 
Table 1: Effect of the target siRNA nanoparticles on invasiveness and metastasis in vivo

\begin{tabular}{|c|c|c|c|c|c|}
\hline & $\begin{array}{c}\text { Mice } \\
(n)\end{array}$ & $\begin{array}{c}\text { peritoneal } \\
\text { dissemination }\end{array}$ & $\begin{array}{l}\text { Retroperitonem } \\
\text { invasion }\end{array}$ & $\begin{array}{c}\text { Liver } \\
\text { metastasis }\end{array}$ & Lung metastasis \\
\hline PBS & 11 & $8 / 11$ & $9 / 11$ & $6 / 11$ & $6 / 11$ \\
\hline Scrambled control siRNA-COL & 10 & $6 / 10$ & $9 / 10$ & $4 / 10$ & $8 / 10$ \\
\hline $\begin{array}{l}\text { Scrambled control siRNA-FA-PEG- } \\
\text { COL nanoparticles }\end{array}$ & 9 & $6 / 9$ & $7 / 9$ & $3 / 9$ & $6 / 9$ \\
\hline $\begin{array}{l}C C D C 88 A \text { siRNA-FA-PEG-COL } \\
\text { nanoparticles }\end{array}$ & 9 & $3 / 9$ & $2 / 9^{\mathrm{a}}$ & $1 / 9$ & $1 / 9^{\mathrm{a}}$ \\
\hline $\begin{array}{l}\text { ARHGEF4 siRNA-FA-PEG-COL } \\
\text { nanoparticles }\end{array}$ & 9 & $4 / 9$ & $3 / 9^{a}$ & $3 / 9$ & $1 / 9^{a}$ \\
\hline $\begin{array}{l}\text { LAMTOR2 siRNA-FA-PEG-COL } \\
\text { nanoparticles }\end{array}$ & 8 & $1 / 8^{\mathrm{a}}$ & $1 / 8^{\mathrm{a}}$ & $2 / 8$ & $2 / 8^{a}$ \\
\hline $\begin{array}{l}m T O R \text { siRNA-FA-PEG-COL } \\
\text { nanoparticles }\end{array}$ & 8 & $2 / 8^{a}$ & $0 / 8^{a}$ & $3 / 8$ & $3 / 8$ \\
\hline $\begin{array}{l}\text { NUP85 siRNA-FA-PEG-COL } \\
\text { nanoparticles }\end{array}$ & 9 & $1 / 9^{a}$ & $1 / 9 \mathrm{a}$ & $1 / 9$ & $3 / 9$ \\
\hline $\begin{array}{l}W A S F 2 \text { siRNA-FA-PEG-COL } \\
\text { nanoparticles }\end{array}$ & 10 & $4 / 10$ & $2 / 10^{\mathrm{a}}$ & $2 / 10$ & $2 / 10^{\mathrm{a}}$ \\
\hline
\end{tabular}

Table 2: Effect of the target siRNA nanoparticles on overall survival in vivo

\begin{tabular}{lcc}
\hline & $\begin{array}{c}\text { Total mice } \\
\text { (n) }\end{array}$ & $\begin{array}{c}\text { Alive mice at } 8 \text { week } \\
\text { post-implantation }\end{array}$ \\
\hline PBS & 10 & $1 / 10$ \\
Scrambled control siRNA-COL & 10 & $1 / 10$ \\
Scrambled control siRNA-FA-PEG-COL nanoparticles & 10 & $1 / 10$ \\
CCDC88A siRNA-FA-PEG-COL nanoparticles & 10 & $1 / 10$ \\
ARHGEF4 siRNA-FA-PEG-COL nanoparticles & 9 & $0 / 9$ \\
LAMTOR2 siRNA-FA-PEG-COL nanoparticles & 10 & $3 / 10^{\mathrm{a}}$ \\
mTOR siRNA-FA-PEG-COL nanoparticles & 10 & $5 / 10^{\mathrm{a}}$ \\
NUP85 siRNA-FA-PEG-COL nanoparticles & 10 & $4 / 10^{\mathrm{a}}$ \\
WASF2 siRNA-FA-PEG-COL nanoparticles & 9 & $1 / 9$ \\
\hline
\end{tabular}

${ }^{\mathrm{a}} p<0.05$ compared to controls (PBS, FA-PEG-COL nanoparticles and scrambled control siRNA)

that prevent cell death or the normal response to drugs and radiotherapy $[20,21]$. The epidermal growth factor receptor (EGFR), which is upstream of $\mathrm{K}$-Ras and involved in the Ras-rapidly accelerated fibrosarcoma (Raf)-MAP kinase kinase (MEK)-extracellular signalregulated kinase (ERK) signaling pathway, plays important roles in PDAC development [22]. EGFR and its ligands are strongly upregulated in PDAC $[23,24]$. The EGFR-targeted agent erlotinib, an oral EGFR tyrosine kinase inhibitor, in combination with gemcitabine has been approved, but provides only marginal benefits [25]. A clinical study (CONKO-005) indicated that adjuvant gemcitabine plus erlotinib does not improve overall survival in patients with R0 PDAC resections [26]. These previous studies indicated that inhibition of K-Ras proto-oncogene GTPase (K-Ras)associated signaling factors may be effective as targeted molecular therapy to inhibit the growth of PDACs; however, their effects are extremely limited. Many papers assessing tumor growth of PDAC have been published, but no essential molecular targets have been identified. The major hallmark of PDAC is its early systemic dissemination and its extraordinary local tumor progression, and a standing problem in therapy for PDAC is metastatic disease [27]. Therefore, agents that target other signaling pathways associated with invasiveness and metastasis in PDAC are needed to improve outcomes of PDAC patients. 
Nucleic acids such as siRNAs have tremendous versatility and target specificity [28]. siRNAs need to be modified in a manner that protects them from enzymatic degradation, thereby improving the proportion of siRNAs that reach tumor tissues after systemic administration. Subsequently, the siRNAs that reach tumor tissues need to be efficiently taken up by tumor cells. We aim to establish a delivery system with a ligand for the FA receptor that is highly expressed in the cytoplasm and membrane of PDAC cells. Intravenous injection of siRNAs against ARHGEF4, CCDC88A, LAMTOR2, mTOR, NUP85, and $W A S F 2$, which were modified with FA to allow binding to the FA receptor and PEG-COL nanoparticles, enabled target siRNA-FA-PEG-COL nanoparticle-mediated
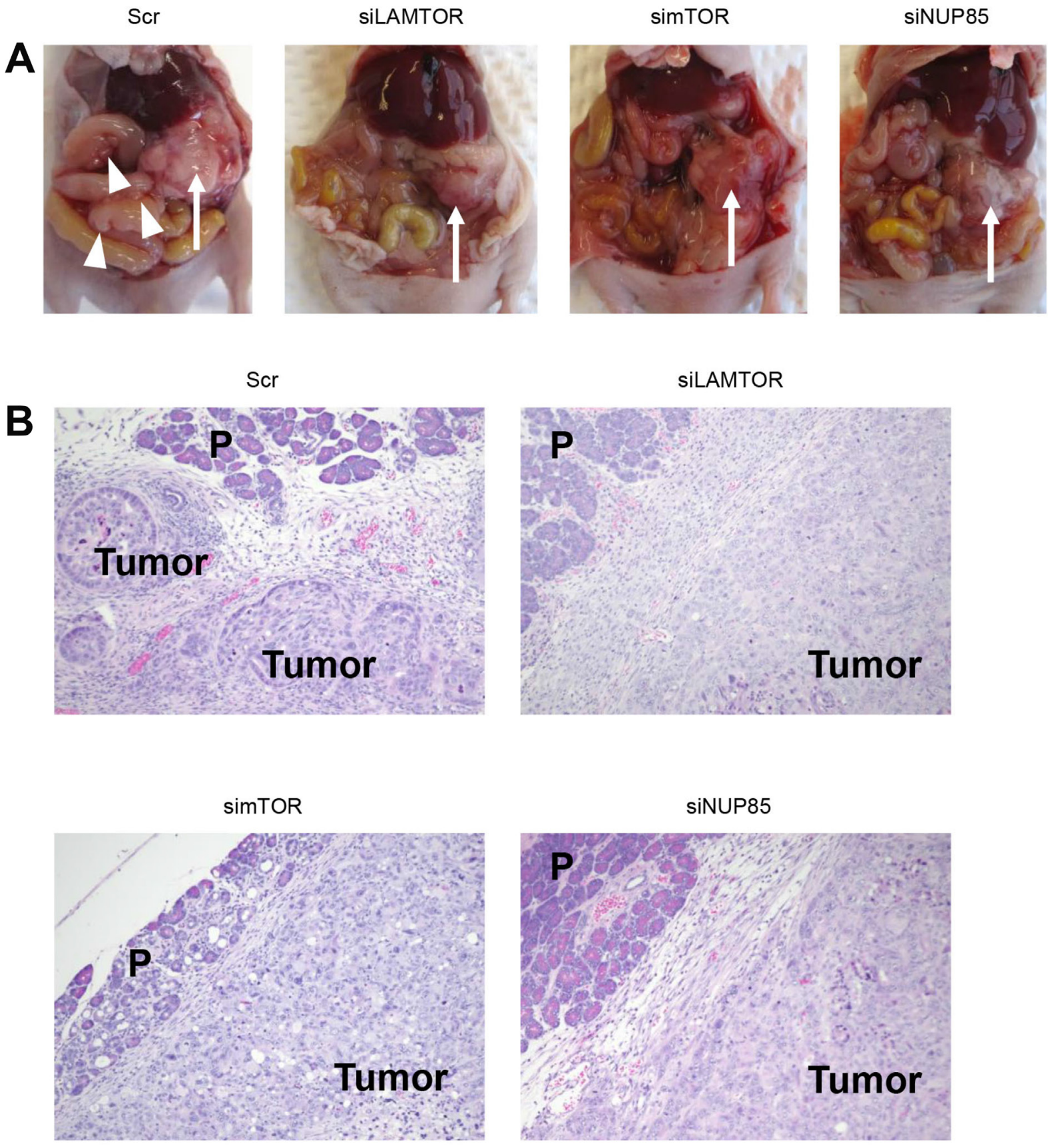

Figure 8: Disease progression in the orthotopic mouse model of PDAC. (A) Representative S2-013-derived PDAC tumor tissues in S2-013 tumor-bearing mice treated with scrambled control siRNA-FA-PEG-COL nanoparticles (Scr) and target siRNA-FAPEG-COL nanoparticles against mRNAs for LAMTOR2 (siLAMTOR2), mTOR (simTOR), and NUP85 (siNUP85). Arrow, primary tumor; arrowheads, dissemination nodules in the abdominal cavity. (B) Hematoxylin and eosin staining of representative sections of S2-013derived PDAC tumor tissues in mice treated with scrambled control siRNA-FA-PEG-COL nanoparticles or target siRNA-FA-PEG-COL nanoparticles against mRNAs for LAMTOR2, mTOR, and NUP85. 

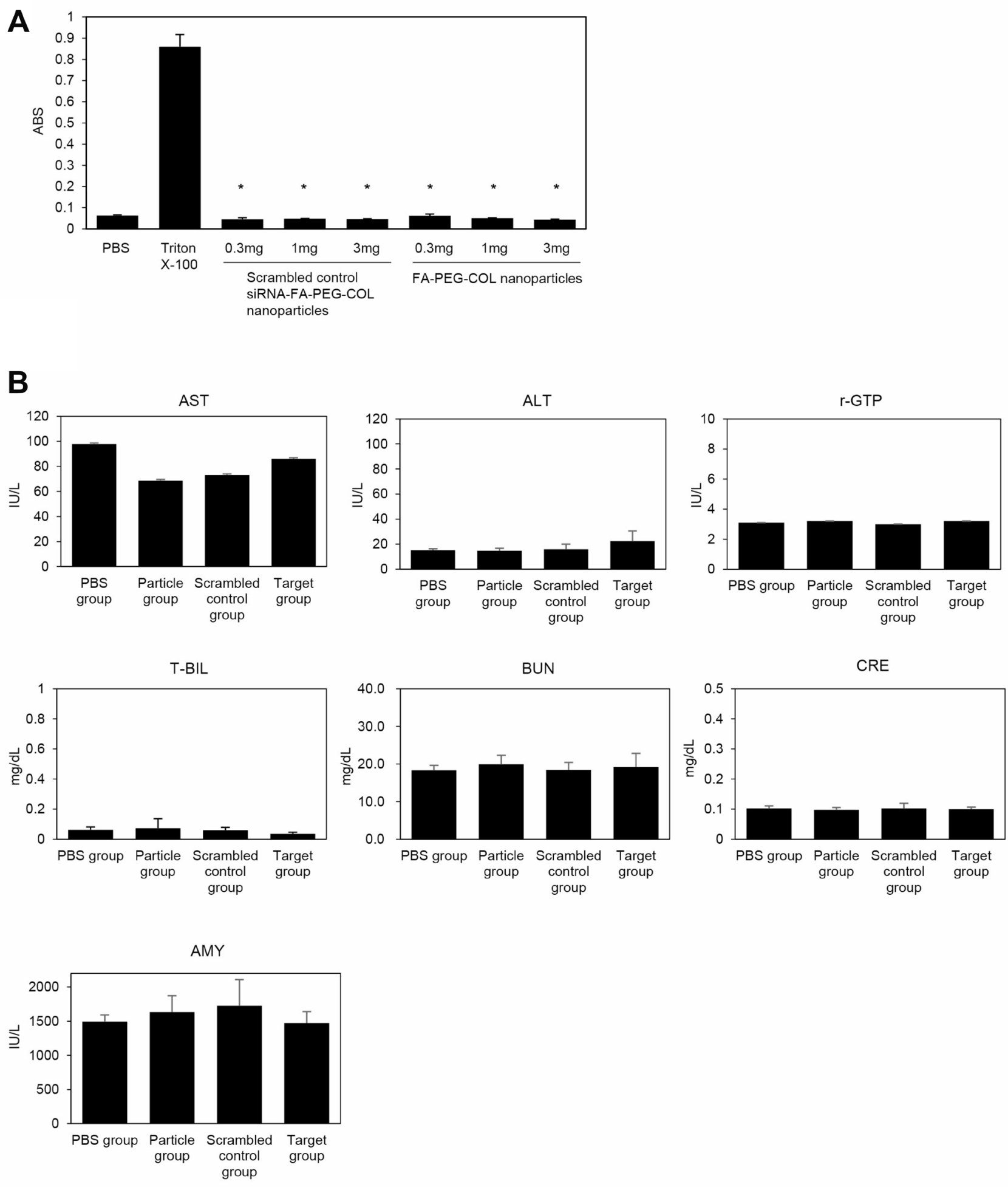

Figure 9: Toxicological study of siRNA-FA-PEG-COL nanoparticles. (A) In vitro hemolysis test. Diluted mouse blood was exposed to FA-PEG-COL nanoparticles and scrambled control siRNA-FA-PEG-COL nanoparticles. PBS was used as the negative control, and Triton X-100 was used as the positive control. Data were derived from three independent experiments. Columns, mean; bars, standard deviation (SD). ${ }^{*} p<0.05$ compared to the positive control (Student's $t$-test). (B) Serum biochemical parameters indicating the functions of liver (ALT, AST, r-GTP, and T-BIL), kidney (BUN, CRE), and pancreas (AMY) 14 days after injection of PBS alone (PBS group), FA-PEG-COL nanoparticles alone (Particle group), scrambled control siRNA-FA-PEG-COL nanoparticles (scrambled control group), or siRNA-FA-PEG-COL nanoparticles against CCDC88A (target group) into mice. 
passive delivery to human PDAC tumor cells and promoted efficient siRNA endocytosis of these siRNA particles via the FA receptor on PDAC cells.

At least 22 RNA interference-based drugs have entered clinical trials, but most siRNA drug delivery systems are still in preclinical studies [29]. Targeted therapies using siRNA nanoparticles have been developed to discover better approaches for patients with other cancers. FA-conjugated BRCAA1 siRNA nanoparticles result in highly efficient siRNA delivery and reduction in the size of gastric cancer xenografts in vivo [30]. 7-PLK1 siRNA nanoparticles injected systemically reduce nonsmall cell lung cancer tumor growth in an orthotopic lung cancer mouse model [31]. Instead of focusing on the growth of PDACs, the present study showed that retroperitoneal invasion and metastasis to the liver and lung from the PDAC tumors in cancer-bearing nude mice were suppressed by administration of target siRNA-FAPEG-COL nanoparticles against ARHGEF4, CCDC88A, LAMTOR2, mTOR, NUP85, and WASF2. The key molecules that induce PDAC tumor progression bypass one signal transduction pathway via another pathway. Thus, new drugs that target different pathways should increase the effectiveness of treatments. High expression of ARHGEF4, CCDC88A, and WASF2 predicts poor prognosis [10-12]. These proteins promote cell invasion by influencing ERK1/2 and Glycogen Synthase Kinase $3 \alpha / \beta$ (GSK-3 $\alpha / \beta)$ signaling [10]; Src, ERK1/2, and AMPactivated protein kinase 1 (AMPK1) signaling [11]; and alpha-actinin 4 (ACTN4) and p27 signaling [12] in pancreatic cancer, respectively. LAMTOR2 is a member of the late endosomal/lysosomal adaptor and LAMTOR complex that regulates mTOR and ERK activation and serves as a convergence point for ERK and mTOR complex 1 (mTORC1) signaling [32]. NUP85 links activated C-C Motif Chemokine Receptor 2 (CCR2) to the phosphatidylinositol 3-kinase (PI3K)-Rac-lamellipodium protrusion cascade [33]. Inhibition of ARHGEF4, CCDC88A, LAMTOR2, mTOR, NUP85, and/or WASF2 may be effective as targeted molecular therapy, because any such therapy is expected to inhibit the invasiveness and metastasis of PDACs by blocking multiple signaling pathways related to these processes. The data presented herein showed that the siRNA-FA-PEG-COL nanoparticles against LAMTOR2, mTOR, and NUP85 strongly inhibited regional invasion of the retroperitoneum and peritoneal dissemination, and that siRNA-FA-PEG-COL nanoparticles against CCDC88A, ARHGEF4, LAMTOR2, and $W A S F 2$ significantly inhibited lung metastasis.

The results from the survival study suggest that in our orthotopic mouse model of PDAC, at least partial inhibition of peritoneal dissemination and retroperitoneal invasion from PDAC tumors occurs due to administration of the siRNA-FA-PEG-COL nanoparticles against LAMTOR2, mTOR, and NUP85, leading to improved survival, compared with mice treated with other target
siRNA-FA-PEG-COL nanoparticles. The siRNA-FAPEG-COL nanoparticles against CCDC88A, ARHGEF4 and $W A S F 2$ significantly inhibited regional invasion of the retroperitoneum and metastasis to lung; however, they did not improve survival. This may be due to the remarkably short overall survival of our orthotopic mouse model of PDAC, and peritoneal dissemination and retroperitoneal invasion may have been a direct cause of death in our orthotopic mouse model. Free PDAC cells in the peritoneal cavity have been observed in $20-40 \%$ of human PDAC cases, even in patients without nodal involvement who underwent early resection [34]. Thus, to improve overall survival in patients with PDAC, more effective and better tolerated therapies that inhibit local and regional invasion from the tumor as well as distant metastasis are needed. The data for the siRNA-FA-PEGCOL nanoparticles against LAMTOR2, mTOR, and NUP85 suggest that these siRNA nanoparticles could be useful for discovering better approaches for patients with PDAC.

Blood examinations from mice that were intravenously injected with siRNA-FA-PEG-COL nanoparticles showed no definite systemic dysfunction of organ systems including liver, kidney, and pancreas. No gross abnormal findings were noted in the lung, liver, kidney, or pancreas. Moreover, the result of the in vitro test showed that mouse erythrocytes were not lysed by FAPEG-COL nanoparticles or scrambled control siRNA-FAPEG-COL nanoparticles. These findings suggest that the siRNA-FA-PEG-COL nanoparticles used in this study are safe and biocompatible.

In conclusion, this study suggests the potential to develop specific siRNA-FA-PEG-COL nanoparticles targeting LAMTOR2, mTOR, and NUP85 to inhibit the invasion and metastasis of PDAC and to improve the prognosis. These siRNA particles were delivered with high efficiency to PDAC cells, became localized in PDAC cells, and potently mediated mRNA downregulation of their targets. No definite toxic effect was noted in vitro or in vivo. This approach holds great potential for a novel therapeutic strategy for the treatment of PDACs.

\section{MATERIALS AND METHODS}

\section{Antibodies}

Anti-ARHGEF4 (55213-1-AP) and anti-NUP85 (19370-1-AP) antibodies were purchased from Proteintech (Chicago, IL, USA). Anti-CCDC88A antibody (MABT100) was purchased from Merck Millipore (Temecula, CA, USA). Anti-LAMTOR2 antibody (8145) was purchased from Cell Signaling (Danvers, MA, USA). Anti-WASF2 (sc-33548) and anti-mTOR (sc-1549) antibodies were purchased from Santa Cruz Biotechnology (Dallas, Texas, USA). Anti-folate receptor alpha antibody (MAB5646) was purchased from R\&D SYSTEMS (Minneapolis, MN, USA). 


\section{Cell culture}

The human PDAC cell line S2-013, which is a subline of SUIT-2, the human PDAC cell line PANC-1, and HPNE immortalized normal pancreatic epithelial cells were maintained in Dulbecco's modified Eagle's medium (Gibco-BRL, Carlsbad, CA, USA) containing 10\% fetal calf serum as published previously [19].

\section{Synthesis of the FA-PEG-COL conjugate}

The FA-PEG-COL conjugate was synthesized as published previously [18].

\section{MALDI-TOF MS spectrometry of the FA-PEG- COL conjugate}

Conjugation of FA to PEG was verified with MALDI-TOF Mass (Bruker autoflex Mass Spectrometer; Bruker Japan, Yokohama, Japan) by APRO Life Science Institute (Naruto, Japan).

\section{Fabrication of nanoparticles}

Fabrication of nanoparticles was carried out as published previously [18].

\section{Preparation of siRNA-loaded COL and FA-PEG- COL nanoparticles}

The siRNAs against ARHGEF4 (5'-CAAGCCAG AAACCACAUUUAA-3'), CCDC88A (5'-AACGUUGG UUACACUACGUGA-3'), LAMTOR2 (5'-CACCGCU GCCAUAGCCAGUAA-3'), mTOR (5'-ACUCGCUGA UCCAAAUGACAA-3'), NUP85 (5'-CAGCGGCAGA UGACUGAACAA-3'), WASF2 (5'-UAGGAUUAGAU CAUUAGCUCA-3'), and a scrambled control siRNA (5'-UUCUCCGAACGUGUCACGUAU-3') were synthesized by GeneDesign (Osaka, Japan). siRNA $(18.2 \mu \mathrm{L} ; 1.38 \mathrm{grams} / \mu \mathrm{L})$ in RNase-free water was added to $500 \mu \mathrm{L}$ of $0.70 \mathrm{mg} / \mathrm{mL}$ thiamine pyrophosphate solution and then added to pre-warmed COL at $62^{\circ} \mathrm{C}$, or $500 \mu \mathrm{L}$ of $6 \mathrm{mg} / \mathrm{mL}$ FA-PEG-COL solution, yielding a thiamine pyrophosphate-to-FA-PEG-COL weight ratio of 1:8.6. The nanoparticles were shaken in the dark for $30 \mathrm{~min}$ and incubated at ambient temperature for $30 \mathrm{~min}$ before use or analysis.

The morphology and particle size of FA-PEG-COL were characterized with SEM (JSM-7200F; JEOL Ltd., Tokyo, Japan) by JEOL Ltd.

\section{Confocal microscopy}

S2-013 and HPNE cells were seeded on glass coverslips in FA-free RPMI 1640 (Sigma-Aldrich, St. Louis, MO, USA) and placed in a cell culture incubator. Cells were allowed to adhere overnight. Scrambled control siRNA was loaded with Alexa 488-conjugated FA-PEG-COL nanoparticles as described in the section called "Preparation of siRNA-loaded COL and FA-PEGCOL nanoparticles". Alexa 488-labeled FA-PEG-COL nanoparticles and Alexa 488-labeled scrambled control siRNA-FA-PEG-COL nanoparticles were added to S2013 and HPNE cells and cultured for $24 \mathrm{~h}$ at $37^{\circ} \mathrm{C}$. The cells were then fixed with $4 \%$ paraformaldehyde, and each specimen was visualized using a Zeiss LSM 510 META microscope (Carl Zeiss, Gottingen, Germany).

\section{Flow cytometric analysis}

S2-013 and HPNE cells $\left(1 \times 10^{6}\right.$ cells $)$ were cultured in 35-mm plates in $2 \mathrm{~mL}$ FA-free RPMI 1640 and placed in a cell culture incubator ( $95 \%$ air, $5 \% \mathrm{CO}_{2}$ at $37^{\circ} \mathrm{C}$ ). Cells were allowed to adhere overnight. Alexa 488-labeled scrambled control siRNA-loaded FA-PEG-COL nanoparticles were prepared as described in the section called "Confocal microscopy". These nanoparticles were added to cultured S2-013 and HPNE cells for $24 \mathrm{~h}$. Cells were washed three times with ice cold PBS, trypsinized, and re-suspended in PBS. Quantitative cellular uptake of nanoparticles was performed using a FACS Calibur flow cytometer (Becton-Dickinson, San Jose, CA, USA).

\section{In vitro gene knockdown efficiency}

In vitro transfection and gene knockdown studies were performed in S2-013 cells. Cells were seeded in a 6 -well plate at a density of $6 \times 10^{6}$ cells per well in 2.0 mL FA-free RPMI 1640 containing 10\% fetal calf serum and incubated for $8 \mathrm{~h}$ before changing to fresh FA-free RPMI 1640. A scrambled control siRNA and the siRNAs targeting mRNAs for ARHGEF4, CCDC88A, LAMTOR2, $m T O R, N U P 85$, and WASF2 were loaded into FA-PEGCOL nanoparticles and added to cultured S2-013 cells for $48 \mathrm{~h}$. Gene knockdown was assessed at two levels, the protein level using SDS-PAGE followed by Western blotting and the mRNA level using semi-quantitative RT-PCR.

\section{Semi-quantitative RT-PCR}

Total RNAs were extracted from each culture of cells using Trizol reagent (Invitrogen Life Technologies, Carlsbad, CA, USA) according to the manufacturer's recommendations, treated with DNase I (Roche Diagnostic, Mannheim, Germany), and reverse transcribed to single-stranded cDNAs using oligodeoxythymidylic acid primers with Superscript II reverse transcriptase (Invitrogen). We prepared appropriate dilutions of each single-stranded cDNA for subsequent PCR amplification by quantitatively monitoring GAPDH as a control. All reactions involved initial denaturation at $94^{\circ} \mathrm{C}$ for $2 \mathrm{~min}$ followed by 21 cycles (for GAPDH) or 28 cycles (for targets) at $94^{\circ} \mathrm{C}$ for $30 \mathrm{sec}, 58^{\circ} \mathrm{C}$ for $30 \mathrm{sec}$, and $72^{\circ} \mathrm{C}$ 
for 1 min on a GeneAmp PCR system 9700 (PE Applied Biosystems, Foster City, CA, USA).

\section{Trans-well motility assay}

The trans-well motility assay using S2-013 cells was carried out as published previously [19]. A scrambled control siRNA and the siRNAs targeting mRNAs for ARHGEF4, CCDC88A, LAMTOR2, mTOR, NUP85, and $W A S F 2$ were loaded into FA-PEG-COL nanoparticles and added to cultured S2-013 cells for $24 \mathrm{~h}$. Cells were plated in BD BioCoat Control Culture Inserts (24-well plates, $8-\mu \mathrm{m}$ pore size; Becton Dickinson, San Jose, CA, USA).

\section{Matrigel invasion assay}

A scrambled control siRNA and the siRNAs targeting mRNAs for ARHGEF4, CCDC88A, LAMTOR2, MTOR, NUP85, and WASF2 were loaded into FA-PEG-COL nanoparticles and added to cultured S2-013 cells for $24 \mathrm{~h}$. A two-chamber invasion assay was used to assess cell invasion (24-well plates, $8-\mu \mathrm{m}$ pore size membrane coated with a layer of Matrigel extracellular matrix proteins; Becton Dickinson) as published previously [19].

\section{Mice and orthotopic implantation of tumor cells}

Our orthotopic mouse model of PDAC was established by surgical implantation of human PDAC cells into the pancreas of an immunocompetent host [19]. Briefly, pathogen-free female athymic nude mice (BALB/ cSlc-nu/nu, 6 weeks of age) were purchased from Japan SLC (Shizuoka, Japan). Mice were treated in accordance with the Institutional Animal Care and Use Committee guidelines of Kochi University. S2-013 cells $\left(8.0 \times 10^{5}\right)$ were surgically and orthotopically implanted into the pancreas of each mouse. Mice were treated with siRNAloaded FA-PEG-COL nanoparticles once per week for six weeks, and then sacrificed 42 days after cell implantation. Sections of PDAC tissues, lung, and liver were prepared, and hematoxylin and eosin staining was then used to determine the presence or absence of tumor invasion into the retroperitoneum and of metastatic lesions in the lung and liver.

\section{In vivo accumulation of FA-PEG-COL nanoparticles loaded with siRNA}

The siRNAs against mRNAs for ARHGEF4, CCDC88A, LAMTOR2, mTOR, NUP85, and WASF2 and scrambled control siRNA-loaded FA-PEG-COL nanoparticles were prepared as described in the section called "Preparation of siRNA-loaded COL and FA-PEGCOL nanoparticles". The siRNA-loaded nanoparticles were collected by centrifugation $(13,000 \times g, 15 \mathrm{~min})$ and re-suspended in $100 \mu \mathrm{L}$ PBS. Mice that had been surgically and orthotopically implanted with S2-013 cells into the pancreas were given via the tail vein a $100 \mu \mathrm{L}$ bolus injection of scrambled control siRNA-COL; FAPEG-COL nanoparticles; siRNAs against mRNAs for ARHGEF4, CCDC88A, LAMTOR2, mTOR, NUP85, and $W A S F 2$; or scrambled control siRNA-loaded FAPEG-COL nanoparticles 4 days after implantation. Each injection of nanoparticles was given once weekly. Forty-one days after implantation, a final injection of nanoparticles labeled with Alexa 594 was given to one mouse per group, and $24 \mathrm{~h}$ later, fluorescence images were captured using an IVIS Spectrum In Vivo Imaging System (PerkinElmer, Waltham, MA, USA) with filters set at kexc $640 \mathrm{~nm}$ and kemi $680 \mathrm{~nm}$. Each mouse was sacrificed 42 days after implantation; hematoxylin and eosin staining was then used to determine the presence or absence of tumor invasion into the retroperitoneum and of metastatic lesions in the lung and liver.

\section{PDAC tumor tissue perfusion}

Twenty-four hours after intravenous injection of Alexa 647-labeled siRNA-FA-PEG-COL nanoparticles via the tail vein into S2-013 tumor-bearing mice, mice were anesthetized, systemically perfused with $0.9 \%$ sodium chloride followed by $10 \mathrm{~mL}$ of $4 \%$ paraformaldehyde, and the S2-013-derived PDAC tumors were removed. Frozen tissue sections of the S2-013-derived PDAC tumors were prepared, and each specimen was visualized using a VKX1000 microscope (Keyence, Osaka, Japan).

\section{Hemolysis assay using siRNA-loaded nanoparticles}

FA-PEG-COL nanoparticles and scrambled control siRNA-FA-PEG-COL nanoparticles were suspended in PBS at concentrations of $0.3,1.0$, and $3.0 \mathrm{mg} / \mathrm{mL}$. Mouse blood was collected by cardiac puncture. Red blood cells $(\mathrm{RBCs})$ were collected by centrifugation $(1500 \times g, 10$ min at $4^{\circ} \mathrm{C}$ ) and diluted 100 times with pre-chilled PBS. RBCs and nanoparticles $(250 \mu \mathrm{L}$ each) were added to a $1.5-\mathrm{mL}$ tube and shaken gently for $1 \mathrm{~min}$. The samples were incubated at $37^{\circ} \mathrm{C}$ for $60 \mathrm{~min}$. The samples were centrifuged $\left(1500 \times \mathrm{g}, 15 \mathrm{~min}\right.$ at $\left.4^{\circ} \mathrm{C}\right)$, and the absorbance of the supernatants was recorded at $541 \mathrm{~nm}$. PBS was used as the negative control, and Triton X-100 (2\% w/v) was used as the $100 \%$ lysis positive control.

\section{Blood test}

Pathogen-free female athymic nude mice (BALB/ cSlc-nu/nu, 8 weeks of age) were given via the tail vein a $100-\mu \mathrm{L}$ bolus injection of FA-PEG-COL nanoparticles, siRNAs against $C C D C 88 A$ mRNA, or scrambled control siRNA-loaded FA-PEG-COL nanoparticles. Nanoparticles were given once weekly. After 6 weeks, each mouse was anesthetized, and terminal blood collection was performed 
from the axillary vessels. After centrifuging at $1500 \times g$ at $4^{\circ} \mathrm{C}$ for $15 \mathrm{~min}$, the cell-free supernatant serum was collected. AST, ALT, $\gamma$-GT, T-BIL, AMY, BUN and CRE levels were used to detect liver, kidney, and pancreas function (FUJIFILM Wako Chemicals, Osaka, Japan).

\section{Statistical analysis}

StatFlex software (Ver6; YUMIT, Osaka, Japan) and SAS software (Ver9.1.3; SAS Institute, Cary, NC, USA) were used for statistical analysis. The significance of differences between groups was determined using the two-tailed Student's $t$-test or Fisher's exact test, as appropriate. Cumulative survival rates were calculated using the Kaplan-Meier method using R (version 3.3.3; The $\mathrm{R}$ Foundation, Wien, Austria). For all analyses, $p<$ 0.05 was considered statistically significant.

\section{Abbreviations}

PDAC: pancreatic ductal adenocarcinoma; siRNA: interfering RNA; FA: folic acid; PEG: polyethylene glycol; COL: chitosan oligosaccharide lactate; SEM: scanning electron microscope; RT-PCR: reverse transcription-PCR; EGFR: epidermal growth factor receptor; Raf: Ras-rapidly accelerated fibrosarcoma; MEK: MAP kinase kinase; ERK: MEK-extracellular signal-regulated kinase; GSK: glycogen synthase kinase; PI3K: phosphatidylinositol 3-kinase.

\section{ACKNOWLEDGMENTS}

We thank Ms. Miki Nishigawa and Ms. Rieko Takahashi for their excellent technical assistance, Mr. Shunichi Manabe for performing immunohistochemical stains, Ms. Sayo Kataoka for performing flow cytometric analysis, and Mr. Atsuya Nobumoto, Mr. Takunori Mogawa, and Dr. Masayuki Tsuda for performing animal experiments. We thank Hajime Kuroiwa for the statistical analysis of the data. We thank Dr. Mutsuo Furihata for thought-provoking discussion and logistical support.

\section{CONFLICTS OF INTEREST}

The authors have declared that no competing interests exist.

\section{FUNDING}

This study was supported by the Center for Clinical and Translational Research of Kyushu University selected by the Japan Agency for Medical Research and Development (AMED) and by Grants-in-Aid for Scientific Research (KAKENHI; 15K14396, 15K08346, 16K09397, and 17K09463).

\section{REFERENCES}

1. Siegel RL, Miller KD, Jemal A. Cancer statistics, 2016. CA Cancer J Clin. 2016; 66:7-30. https://doi.org/10.3322/ caac.21332. [PubMed]

2. Hidalgo M. Pancreatic cancer. N Engl J Med. 2010; 362:1605-1617. https://doi.org/10.1056/NEJMra0901557. [PubMed]

3. Philip PA. First- and second-line treatment of metastatic pancreatic adenocarcinoma: the conundrum continues. Gastrointest Cancer Res. 2009; 3:37-39. [PubMed]

4. Abbruzzese JL, Hess KR. New option for the initial management of metastatic pancreatic cancer? J Clin Oncol. 2014; 32:2405-2407. https://doi.org/10.1200/ JCO.2013.54.4155. [PubMed]

5. Von Hoff DD, Ervin T, Arena FP, Chiorean EG, Infante J, Moore M, Seay T, Tjulandin SA, Ma WW, Saleh MN, Harris M, Reni M, Dowden S, et al. Increased survival in pancreatic cancer with nab-paclitaxel plus gemcitabine. N Engl J Med. 2013; 369:1691-1703. https://doi.org/10.1056/ NEJMoa1304369. [PubMed]

6. Biankin AV, Waddell N, Kassahn KS, Gingras MC, Muthuswamy LB, Johns AL, Miller DK, Wilson PJ, Patch AM, Wu J, Chang DK, Cowley MJ, Gardiner BB, et al. Pancreatic cancer genomes reveal aberrations in axon guidance pathway genes. Nature. 2012; 491:399-405. https://doi.org/10.1038/nature11547. [PubMed]

7. Warshaw AL, Fernández-del Castillo C. Pancreatic carcinoma. N Engl J Med. 1992; 326:455-465. https://doi. org/10.1056/NEJM199202133260706. [PubMed]

8. Taniuchi K, Furihata M, Hanazaki K, Saito M, Saibara T. IGF2BP3-mediated translation in cell protrusions promotes cell invasiveness and metastasis of pancreatic cancer. Oncotarget. 2014; 5:6832-6845. https://doi.org/10.18632/ oncotarget.2257. [PubMed]

9. Taniuchi K, Furihata M, Saibara T. KIF20A-mediated RNA granule transport system promotes the invasiveness of pancreatic cancer cells. Neoplasia. 2014; 16:1082-1093. https://doi.org/10.1016/j.neo.2014.10.007. [PubMed]

10. Taniuchi K, Furihata M, Naganuma S, Saibara T. ARHGEF4 predicts the poor prognosis and promotes cell invasion by influencing ERK1/2 and GSK-3 $\alpha / \beta$ signaling in pancreatic cancer. Int J Oncol. 2018; 53:2224-2240. https://doi. org/10.3892/ijo.2018.4549. [PubMed]

11. Tanouchi A, Taniuchi K, Furihata M, Naganuma S, Dabanaka K, Kimura M, Watanabe R, Kohsaki T, Shimizu T, Saito M, Hanazaki K, Saibara T. CCDC88A, a prognostic factor for human pancreatic cancers, promotes the motility and invasiveness of pancreatic cancer cells. J Exp Clin Cancer Res. 2016; 35:190. https://doi.org/10.1186/s13046016-0466-0. [PubMed]

12. Taniuchi K, Furihata M, Naganuma S, Saibara T. WAVE2 is associated with poor prognosis in pancreatic cancers and promotes cell motility and invasiveness via binding 
to ACTN4. Cancer Med. 2018; 7:5733-5751. https://doi. org/10.1002/cam4.1837. [PubMed]

13. Nakayama T, Butler JS, Sehgal A, Severgnini M, Racie T, Sharman J, Ding F, Morskaya SS, Brodsky J, Tchangov L, Kosovrasti V, Meys M, Nechev L, et al. Harnessing a physiologic mechanism for siRNA delivery with mimetic lipoprotein particles. Mol Ther. 2012; 20:1582-1589. https://doi.org/10.1038/mt.2012.33. [PubMed]

14. Creusat G, Thomann JS, Maglott A, Pons B, Dontenwill M, Guérin E, Frisch B, Zuber G. Pyridylthiourea-grafted polyethylenimine offers an effective assistance to siRNAmediated gene silencing in vitro and in vivo. J Control Release. 2012; 157:418-426. https://doi.org/10.1016/j. jconrel.2011.10.007. [PubMed]

15. Wang J, Lu Z, Wientjes MG, Au JL. Delivery of siRNA therapeutics: barriers and carriers. AAPS J. 2010; 12:492503. https://doi.org/10.1208/s12248-010-9210-4. [PubMed]

16. Shi J, Zhang H, Wang L, Li L, Wang H, Wang Z, Li Z, Chen C, Hou L, Zhang C, Zhang Z. PEI-derivatized fullerene drug delivery using folate as a homing device targeting to tumor. Biomaterials. 2013; 34:251-261. https://doi. org/10.1016/j.biomaterials.2012.09.039. [PubMed]

17. Gao W, Xiang B, Meng TT, Liu F, Qi XR. Chemotherapeutic drug delivery to cancer cells using a combination of folate targeting and tumor microenvironment-sensitive polypeptides. Biomaterials. 2013; 34:4137-4149. https:// doi.org/10.1016/j.biomaterials.2013.02.014. [PubMed]

18. Li TS, Yawata T, Honke K. Efficient siRNA delivery and tumor accumulation mediated by ionically cross-linked folic acid-poly(ethylene glycol)-chitosan oligosaccharide lactate nanoparticles: for the potential targeted ovarian cancer gene therapy. Eur J Pharm Sci. 2014; 52:48-61. https://doi. org/10.1016/j.ejps.2013.10.011. [PubMed]

19. Taniuchi K, Nishimori I, Hollingsworth MA. Intracellular CD24 inhibits cell invasion by posttranscriptional regulation of BART through interaction with G3BP. Cancer Res. 2011; 71:895-905. https://doi.org/10.1158/0008-5472.CAN-102743. [PubMed]

20. Borja-Cacho D, Jensen EH, Saluja AK, Buchsbaum DJ, Vickers SM. Molecular targeted therapies for pancreatic cancer. Am J Surg. 2008; 196:430-441. https://doi. org/10.1016/j.amjsurg.2008.04.009. [PubMed]

21. Ryan DP, Hong TS, Bardeesy N. Pancreatic adenocarcinoma. N Engl J Med. 2014; 371:1039-1049. https://doi.org/10.1056/NEJMra1404198. [PubMed]

22. Gysin S, Lee SH, Dean NM, McMahon M. Pharmacologic inhibition of RAF-->MEK-->ERK signaling elicits pancreatic cancer cell cycle arrest through induced expression of p27Kip1. Cancer Res. 2005; 65:4870-4880. https://doi.org/10.1158/0008-5472.CAN-04-2848. [PubMed]

23. Fjällskog ML, Lejonklou MH, Oberg KE, Eriksson BK, Janson ET. Expression of molecular targets for tyrosine kinase receptor antagonists in malignant endocrine pancreatic tumors. Clin Cancer Res. 2003; 9:1469-1473. [PubMed]
24. Ferrari D, Foa P. Targeted therapies: cui prodest? J Clin Oncol. 2007; 25:4691-4692. https://doi.org/10.1200/ JCO.2007.12.7613. [PubMed]

25. Peréz-Soler R, Saltz L. Cutaneous adverse effects with HER1/EGFR-targeted agents: is there a silver lining? J Clin Oncol. 2005; 23:5235-5246. https://doi.org/10.1200/ JCO.2005.00.6916. [PubMed]

26. Sinn M, Bahra M, Liersch T, Gellert K, Messmann H, Bechstein W, Waldschmidt D, Jacobasch L, Wilhelm M, Rau BM, Grützmann R, Weinmann A, Maschmeyer G, et al. CONKO-005: Adjuvant Chemotherapy With Gemcitabine Plus Erlotinib Versus Gemcitabine Alone in Patients After R0 Resection of Pancreatic Cancer: A Multicenter Randomized Phase III Trial. J Clin Oncol. 2017; 35:33303337. https://doi.org/10.1200/JCO.2017.72.6463. [ubMed]

27. DiMagno EP, Reber HA, Tempero MA. AGA technical review on the epidemiology, diagnosis, and treatment of pancreatic ductal adenocarcinoma. American Gastroenterological Association. Gastroenterology. 1999; 117:1464-1484. https://doi.org/10.1016/S00165085(99)70298-2. [PubMed]

28. Aagaard L, Rossi JJ. RNAi therapeutics: principles, prospects and challenges. Adv Drug Deliv Rev. 2007; 59:7586. https://doi.org/10.1016/j.addr.2007.03.005. [PubMed]

29. Xu CF, Wang J. Delivery systems for siRNA drug development in cancer therapy. Asian Journal of Pharmaceutical Sciences. 2015; 10:1-12. https://doi. org/10.1016/j.ajps.2014.08.011.

30. Cui D, Zhang C, Liu B, Shu Y, Du T, Shu D, Wang K, Dai F, Liu Y, Li C, Pan F, Yang Y, Ni J, et al. Regression of Gastric Cancer by Systemic Injection of RNA Nanoparticles Carrying both Ligand and siRNA. Sci Rep. 2015; 5:10726. https://doi.org/10.1038/srep10726. [PubMed]

31. McCarroll JA, Dwarte T, Baigude H, Dang J, Yang L, Erlich RB, Kimpton K, Teo J, Sagnella SM, Akerfeldt MC, Liu J, Phillips PA, Rana TM, et al. Therapeutic targeting of polo-like kinase 1 using RNA-interfering nanoparticles (iNOPs) for the treatment of non-small cell lung cancer. Oncotarget. 2015; 6:12020-12034. https://doi.org/10.18632/ oncotarget.2664. [PubMed]

32. Bar-Peled L, Schweitzer LD, Zoncu R, Sabatini DM. Ragulator is a GEF for the rag GTPases that signal amino acid levels to mTORC1. Cell. 2012; 150:1196-1208. https:// doi.org/10.1016/i.cell.2012.07.032. [PubMed]

33. Terashima Y, Onai N, Murai M, Enomoto M, Poonpiriya V, Hamada T, Motomura K, Suwa M, Ezaki T, Haga T, Kanegasaki S, Matsushima K. Pivotal function for cytoplasmic protein FROUNT in CCR2-mediated monocyte chemotaxis. Nat Immunol. 2005; 6:827-835. https://doi. org/10.1038/ni1222. [PubMed]

34. Matsuno S, Egawa S, Arai K. Trends in treatment for pancreatic cancer. J Hepatobiliary Pancreat Surg. 2001; 8:544-548. https://doi.org/10.1007/s005340100023. [PubMed] 